\title{
Cannabis against chronic musculoskeletal pain: a scoping review on users and their perceptions
}

Daniela Furrer ${ }^{1,2}$, Edeltraut Kröger ${ }^{1,2,3,4^{*}}$ D , Martine Marcotte ${ }^{1}$, Nathalie Jauvin ${ }^{1}$, Richard Bélanger ${ }^{3,5}$, Mark Ware ${ }^{6}$, Guillaume Foldes-Busque ${ }^{7,8}$, Michèle Aubin ${ }^{1,2,5}$, Pierre Pluye ${ }^{6}$ and Clermont E. Dionne $e^{1,2,5}$

\begin{abstract}
Background: Chronic musculoskeletal pain (CMP) may lead to reduced physical function and is the most common cause of chronic non-cancer pain. Currently, the pharmacotherapeutic options against CMP are limited and frequently consist of pain management with non-steroidal anti-inflammatories, gabapentinoids, or opioids, which carry major adverse effects. Although the effectiveness of medical cannabis (MC) for CMP still lacks solid evidence, several patients suffering from it are exploring this therapeutic option with their physicians.

Objectives: Little is known about patients' perceptions of their MC treatment for CMP. We aimed to increase this knowledge, useful for healthcare professionals and patients considering this treatment, by conducting a scoping literature review, following guidance by Arksey and O'Malley, to describe the views and perceptions of adult patients who had consumed MC to relieve chronic CMP.

Methods: Databases (PUBMED, EMBASE, Web of Science) and websites were searched using combinations of controlled and free vocabulary. All studies and study designs reporting on patients' perceptions regarding MC against CMP were considered. Studies had to include adult patients reporting qualitatively or quantitatively, i.e., through questionnaires, on MC use to treat CMP or other non-cancer pain, since studies reporting exclusively on perceptions regarding CMP were very rare. Study characteristics were extracted and limitations of the study quality were assessed. The review includes patients' demographic characteristics, patterns of MC use, perceived positive and negative effects, use of alcohol or other drugs, reported barriers to CM use, and funding sources of the studies.

Results: Participants of the 49 included studies reported that MC use helped them to reduce CMP and other chronic non-cancer pain, with only minor adverse effects, and some reported improved psychological well-being. In the included studies, men represent between 18 and $88 \%$ of the subjects. The mean age of participants in these studies (42/49) varied between 28.4 and 62.8 years old. The most common route of administration is inhalation.
\end{abstract}

\footnotetext{
* Correspondence: Edeltraut.Kroger.ciussscn@ssss.gouv.qc.ca

${ }^{1}$ Centre d'excellence sur le vieillissement de Québec (CEVQ), Centre Intégré

Universitaire de Santé et de Services Sociaux de la Capitale-Nationale

(CIUSSSCN), Québec, QC, Canada

${ }^{2}$ Centre de Recherche du CHU de Québec-Université Laval, Québec, QC,

Canada

Full list of author information is available at the end of the article
}

(c) The Author(s). 2021 Open Access This article is licensed under a Creative Commons Attribution 4.0 International License, which permits use, sharing, adaptation, distribution and reproduction in any medium or format, as long as you give appropriate credit to the original author(s) and the source, provide a link to the Creative Commons licence, and indicate if changes were made. The images or other third party material in this article are included in the article's Creative Commons licence, unless indicated otherwise in a credit line to the material. If material is not included in the article's Creative Commons licence and your intended use is not permitted by statutory regulation or exceeds the permitted use, you will need to obtain permission directly from the copyright holder. To view a copy of this licence, visit http://creativecommons.org/licenses/by/4.0/. 
Conclusion: MC users suffering from CMP or other chronic non-cancer pain perceived more benefits than harms. However, the information from these studies has several methodological limitations and results are exploratory. These user-reported experiences must thus be examined by well-designed and methodologically sound clinical or observational studies, particularly regarding CMP, where reports are very scarce.

Keywords: Medical cannabis, Musculoskeletal pain, Chronic pain, Non-cancer chronic pain, Perceived effects, Adverse effect

\section{Background}

\section{Musculoskeletal pain}

Musculoskeletal pain is a condition affecting bones, muscles, ligaments, and joints, resulting from underlying diseases or health problems such as osteoarthritis, inflammatory rheumatic diseases, and fibromyalgia, although in many cases the exact cause cannot be identified (Arthritis Society, 2015a). Musculoskeletal pain is the most common type of severe long-term pain and it impacts on all aspects of life by typically affecting dexterity and mobility, and by limiting work and activities of daily living (Woolf et al., 2012). It has been recently reported that one in two American adults lives with a musculoskeletal disease (Yelin et al., 2016), and in Canada, approximately $17 \%$ of the adult population are affected, nearly half of whom (44\%) are aged 65 years or older (Arthritis Society, 2015a). Some cases of musculoskeletal pain are of short duration and have no longterm consequences. Chronic musculoskeletal pain (CMP), which persists for more than 3 months (Task Force on Taxonomy of the International Association for the Study of Pain, 1994), however, is associated with a range of problems such as sleep disorders, depression, anxiety, fatigue, reduced quality of life, and inability to work or socialize (Moore et al., 2014). In the USA, the impact of CMP on the economy in terms of healthcare costs and lost productivity is estimated at US \$304 billion for the year 2013 (Yelin et al., 2016).

Effective pharmacological therapeutic options for the relief of CMP are limited and the treatment remains suboptimal for many patients (Fitzcharles et al., 2016). Examples for this are the use of non-steroidal antiinflammatories, gabapentinoids (e.g., pregabalin and gabapentin), or the antidepressants duloxetine and milnacipran, which have shown clinical efficacy in the treatment of fibromyalgia and may have benefit in osteoarthritis and low back pain. However, it is estimated that only about one-third of patients will have at least $50 \%$ pain relief with one of these agents used as monotherapy; due to significant adverse effects, patients often fail to achieve recommended doses, further diminishing the medications' effectiveness (Goldenberg et al., 2011). Opioids are also used to manage CMP, although the effectiveness of this approach remains uncertain (Petzke \& Enax-Krumova, 2016; Schaefert et al., 2015) and the clinical management of CMP with opioids is challenging due to adverse effects such as dependence and/or addiction leading to possible overdose and death (Atluri et al., 2014; Ballantyne, 2015; Hauser et al., 2016; Tobin et al., 2016). It is therefore urgent to explore new treatment options to relieve pain in persons affected by CMP and thus improve their quality of life and social participation (Rowe \& Caprio, 2013; Gereau et al., 2014; Lynch \& Ware, 2015). Many persons for whom CMP is not satisfactorily relieved are turning to alternative therapies. Among these, the products derived from cannabis are perceived as an interesting analgesic option, both by some physicians and some patients (Elikottil et al., 2009; Boehnke et al., 2016), although its use remains controversial (Hosking \& Zajicek, 2008; D'Souza \& Ranganathan, 2015).

\section{Cannabis and cannabinoids}

The Cannabis sativa plant contains over 100 cannabinoids (ElSohly \& Gul, 2014). The most abundant cannabinoid, delta-9-tetrahydrocannabinol (THC), is responsible for the main psychoactive effect of cannabis, but preclinical studies suggest that THC also has some analgesic and anti-inflammatory effects (Ashton, 2007). The second most abundant cannabinoid, cannabidiol (CBD), has antipsychotic effects and is not intoxicating (Niesink \& van Laar, 2013; Zhu et al., 2006). Preclinical studies also support anti-inflammatory and analgesic effects of this compound (Burstein, 2015; Costa et al., 2007; Maione et al., 2011). The quantities and proportions of the different cannabinoids vary between different sources and preparations of cannabis (Ashton, 2001; de Meijer, 2014). Furthermore, there are differences between herbal preparations and consumption methods of cannabis regarding levels of individual cannabinoids, and between patients regarding the pharmacokinetics of these molecules (MacCallum \& Russo, 2018). These differences affect treatment experiences (i.e., anxiety compared to relaxation), making it hard to come up with evidence-based information to guide physicians and patients on the most appropriate prescribing and dosing of cannabis for a given case (Beaulieu et al., 2016; Ko et al., 2016). Worldwide, several cannabinoid-based medicines are available in several countries. The first product, nabiximols (tradename Sativex ${ }^{\ominus}$, contains the 
cannabinoids THC and CBD. The most common indication for its use is spasticity associated with multiple sclerosis. The second product, nabilone (tradename Cesamet $^{\circ}$ ) contains a synthetic cannabinoid similar to THC and is used to alleviate nausea and vomiting associated with chemotherapy treatments. The third product, dronabinol (tradename $\mathrm{Marinol}^{\circ}$ ), is a synthetic cannabinoid chemically identical to THC and its main indications are anorexia associated with weight loss in patients with AIDS, as well as severe nausea and vomiting caused by cancer chemotherapy (Abuhasira et al., 2018). Quite recently, a product containing cannabidiol, Epidiolex ${ }^{\circ}$, has been approved by the US Food and Drug Administration for the treatment of Dravet syndrome and Lennox-Gastaut syndrome, which are severe epileptic encephalopathies.

\section{Medical cannabis and musculoskeletal pain: gaps in knowledge}

Given the confusion between the terms cannabis, cannabinoids, and cannabis for medical purposes, we will refer to the term "medical cannabis" (MC) in this review, in order to describe cannabis products (plant-based products or pharmaceutical products) used for CMP or other non-cancer chronic pain. Chronic pain in general, including CMP, is the most common reason given for the therapeutic use of MC among adults (Fitzcharles et al., 2016; Swift et al., 2005; Ware et al., 2005; Aggarwal et al., 2009; Arthritis Society, 2015b). The effectiveness of $\mathrm{MC}$ in the management of such pain, however, remains controversial. In a systematic review and metaanalysis on cannabinoids for medical use by Whiting et al., only 4 of the 79 trials included were judged at low risk of bias (Whiting et al., 2015). Individual studies suggested improvement in pain intensity, but most of the differences did not reach clinical significance and there was no clear evidence for an effect of the type of cannabinoid or the mode of administration. It is also important to note that different products were used in the individual studies, plant based or pharmaceutical, making comparisons between the studies even more difficult. Moreover, none of the studies assessed the long-term effects of cannabinoids.

In 2015, Lynch et al. published a systematic review of randomized controlled trials published since 2010 and examining cannabinoids for the treatment of chronic non-cancer pain, including CMP. They reported that seven out of the 11 included studies demonstrated a significant analgesic effect. Several trials also demonstrated improvement in secondary outcomes (e.g., sleep, muscle stiffness, and spasticity) (Lynch \& Ware, 2015). Adverse effects most frequently reported, such as fatigue and dizziness, were mild to moderate in severity and generally well tolerated.
In 2017, the National Academies for Science, Engineering, and Medicine of the USA published an exhaustive review on the health effects of cannabis and cannabinoids and concluded that "there is conclusive or substantial evidence that cannabis or cannabinoids are effective for the treatment of chronic pain in adults", based on a review of reviews, following the conclusions of Whiting et al. (Whiting et al., 2015), as well as two primary studies (National Academies of Sciences E, and Medicine, 2017). It should be pointed out, however, that the conclusions reported in the paper of Whiting et al. should be regarded with caution, as most of the studies assessed in this systematic review showed a high risk of bias.

In 2018, Stockings et al. performed another systematic review and meta-analysis of 47 randomized controlled studies and 57 observational studies on cannabinoids for the treatment of chronic non-cancer pain and concluded that the evidence for the effectiveness of $\mathrm{MC}$ on chronic non-cancer pain is limited [pooled events rates for $50 \%$ reduction in pain were not significant: $18.2 \%$ (cannabinoids) vs $14.4 \%$ (placebo); moreover, the number needed to treat was high $(\mathrm{NNT}=24 ; 95 \% \mathrm{CI}: 15-61)$ and the number needed to harm was low $(\mathrm{NNH}=6$; $95 \% \mathrm{CI}$ : 5 8)]. From the results of the reviewed studies, the authors considered it unlikely that cannabinoids would become an important treatment option in chronic non-cancer pain (Stockings et al., 2018). Similarly, Nugent et al. reported in their 2017 review that the utilization of MC to alleviate chronic pain might be associated with several harms, including increased risk for motor vehicle accidents, psychotic symptoms, and short-term cognitive impairment, in addition to negative impacts on the respiratory tract (Nugent et al., 2017).

Thus, available evidence on the effectiveness of MC against CMP and other chronic non-cancer pain remains limited and the results of systematic reviews are somewhat inconclusive. It is even more difficult to conclude about the use of cannabis specifically in the management of CMP because, according to three systematic reviews of clinical trials on cannabis (Fitzcharles et al., 2016; Stockings et al., 2018), only two clinical trials have focused exclusively on musculoskeletal conditions. The authors of these clinical trials reported that cannabinoids (nabilone or Sativex ${ }^{\circ}$ ) led to a significant decrease in some aspects of pain in patients with fibromyalgia (Skrabek et al., 2008) or rheumatoid arthritis (Blake et al., 2006). However, only a small number of patients were studied for a short period of time in these trials and further methodological limitations may have affected their quality (Aviram \& Samuelly-Leichtag, 2017) (Fitzcharles et al., 2016; Stockings et al., 2018). In conclusion, more high-quality randomized controlled trials comparing herbal cannabis or pharmaceutical cannabinoids with 
established therapies or placebo are necessary to define their role in the management of CMP or other chronic pain (Fitzcharles et al., 2016).

Although the use of MC remains controversial, it is gaining popularity and legal frameworks for its use are increasingly seen under certain conditions in a growing number of countries, i.e., Australia, France, Israel, the Netherlands, the UK, New Zealand, Spain, Germany, 29 US states, and since 1999 in Canada (Aguilar et al., 2018), where "serious arthritis" was mentioned as one of the main diagnoses justifying a license to obtain cannabis for medical use in 2013 (Arthritis Society, 2015b). Several countries are therefore already confronted with increasing use of MC against CMP, including selfmedication, even though its efficacy and safety are still unknown.

Two recent reviews reported on MC use in patients suffering from different diseases, including anxiety, depression, HIV/AIDS, pain, and multiple sclerosis, highlighting that pain is the most frequent reason for $\mathrm{MC}$ use and its increasing frequency in general and cannabis self-medication in particular (Kosiba et al., 2019; Park \& Wu, 2017). However, we did not identify major reviews on the characteristics, motivations, perceptions, and expectations of patients with regard to the use of medical cannabis against musculoskeletal or other chronic non-cancer pain. Thus, a knowledge gap exists in our understanding of patients' characteristics and perceptions with regard to this use. Therefore, we conducted a scoping review to explore and describe these characteristics and perceptions of persons using $\mathrm{MC}$ against chronic non-cancer pain, including CMP. This review represents a first step towards a larger research program on this topic.

\section{Methods}

\section{Eligibility criteria and selection of articles}

The study protocol was submitted to the funding organizations and can be accessed through the corresponding author. Included studies had to comprise adults having used cannabis or cannabinoids for therapeutic purposes, including CMP or other chronic pain. Moreover, study samples had to have included at least several participants with chronic musculoskeletal or non-cancer pain. Qualitative, quantitative, and mixed methods studies were considered.

Studies that were specific to only one disease, other than musculoskeletal conditions or chronic non-cancer pain, such as HIV/AIDS, cancer, multiple sclerosis, epilepsy, inflammatory bowel disease, glaucoma, Tourette's syndrome, neuropathic pain, spinal cord injury, migraine, post-traumatic stress disorder, dementia, or mental illness, as well as palliative care, were excluded. Furthermore, all studies that did not report any patient perceptions or results-including clinical trials on the therapeutic or adverse effects of cannabis-were excluded. Books, meeting abstracts, editorials, letters, policy evaluations, or newspaper articles were also excluded. Initial eligibility was assessed by screening the titles and abstracts of retrieved references by three persons Daniela Furrer, Martine Marcotte, and Norma Perez. Then, full texts of eligible references were reviewed by three persons (Daniela Furrer, Martine Marcotte, and Rosa Martins). Included publications that reported about one study in two or more articles were combined into a single study, with one exception (see below). Thereafter, reference lists of relevant reviews and of included studies were hand searched for additional references following the same procedure.

\section{Information sources}

Three large databases (MEDLINE, EMBASE, and Web of Science) were searched using keywords from the controlled vocabulary and free text, and combined to identify publications on users of cannabis for therapeutic purposes (see search strategies in Appendix 1). The searches were conducted during the second half of 2016, updated in June 2019, and were restricted to publications in English, French, or German with no other time limit.

\section{Search strategy}

This scoping review followed guidance by Arksey and O'Malley, Levac et al., and Colquhoun et al. (Arksey \& O'Malley, 2005; Levac et al., 2010; Colquhoun et al., 2014) and examined the published knowledge regarding perceptions and experiences of MC users suffering from CMP or chronic non-cancer pain. Early search results revealed the scarcity of publications studying $\mathrm{MC}$ users for CMP specifically, and since CMP represents the most common etiology for chronic non-cancer pain, we expanded our search to all studies including patients using $\mathrm{MC}$ for chronic non-cancer pain (Podichetty et al., 2003). Moreover, given the scarcity of studies on the perceptions of users of $\mathrm{MC}$, we decided to include both plant-based products and pharmaceutical products such as nabilone or nabiximols in the present review, similarly to some of the included studies (Hazekamp et al., 2013). As such, in the remainder of the manuscript, the abbreviation $\mathrm{MC}$ refers to both plant-based products and cannabis-derived medicine.

\section{Data collection and quality appraisal}

For this narrative synthesis, the following data were extracted by three persons into pre-determined Word files (Daniela Furrer, Martine Marcotte, and Rosa Martins) from the included studies: study design and setting, period of data collection, sample size, participants' age 
and sex, indications for MC consumption, patterns of MC use, perceived benefits and adverse effects of use, and financial support for the study. When available, MC consumption as a substitute for other drugs, as well as barriers to $\mathrm{MC}$ use, were also documented. No individual quality appraisal was performed, according to the guidance used (Arksey \& O'Malley, 2005; Levac et al., 2010; Colquhoun et al., 2014), but multiple limitations of the included study designs are outlined in the discussion.

\section{Results}

A total of 3639 references were first identified, and the full-text was screened for 201 articles, of which 52 publications reporting on 49 studies met the inclusion criteria (Fig. 1). In one publication (Perron et al., 2015), a subsample from a previous study (Ilgen et al., 2013) was used but, since study objectives and measures were different, they were treated as two different studies.

\section{Characteristics of the included studies}

The main characteristics of all included studies are summarized in Fig. 2.
Among all included studies, only two examined the prevalence of cannabis use exclusively among patients suffering from CMP (Ste-Marie et al., 2016). Most of the studies focused on mixed samples that included patients with CMP (between 2 and 91\% of participants) (31 studies) (Swift et al., 2005; Aggarwal et al., 2009; Hazekamp et al., 2013; Ilgen et al., 2013; Aggarwal et al., 2013a; Aggarwal et al., 2013b; Belle-Isle et al., 2014; Bottorff et al., 2011; Bruce et al., 2018; Coomber et al., 2003; Degenhardt et al., 2015; Erkens et al., 2005; Gorter et al., 2005; Haroutounian et al., 2016; Harris et al., 2000; Hoffman et al., 2017; Kilcher et al., 2017; Lucas \& Walsh, 2017; Lynch et al., 2006; Nunberg et al., 2011; Ogborne et al., 2000; Pedersen \& Sandberg, 2013; Piper et al., 2017; Reinarman et al., 2011; Schnelle et al., 1999; Sexton et al., 2016; Shiplo et al., 2016; Ste-Marie et al., 2012; Troutt \& DiDonato, 2015; Walsh et al., 2013; Ware et al., 2003) or experiencing unspecified chronic non-cancer pain (between 24 and $97 \%$ of participants) (17 studies) (Boehnke et al., 2016; Perron et al., 2015; Alexandre, 2011; Bonn-Miller et al., 2014; Brunt et al., 2014; Corroon Jr. et al., 2017; Cranford et al., 2016; Crowell, 2017; Fanelli et al., 2017; Grella et al., 2014; Grotenhermen \& Schnelle, 2003; Hazekamp \& Heerdink,

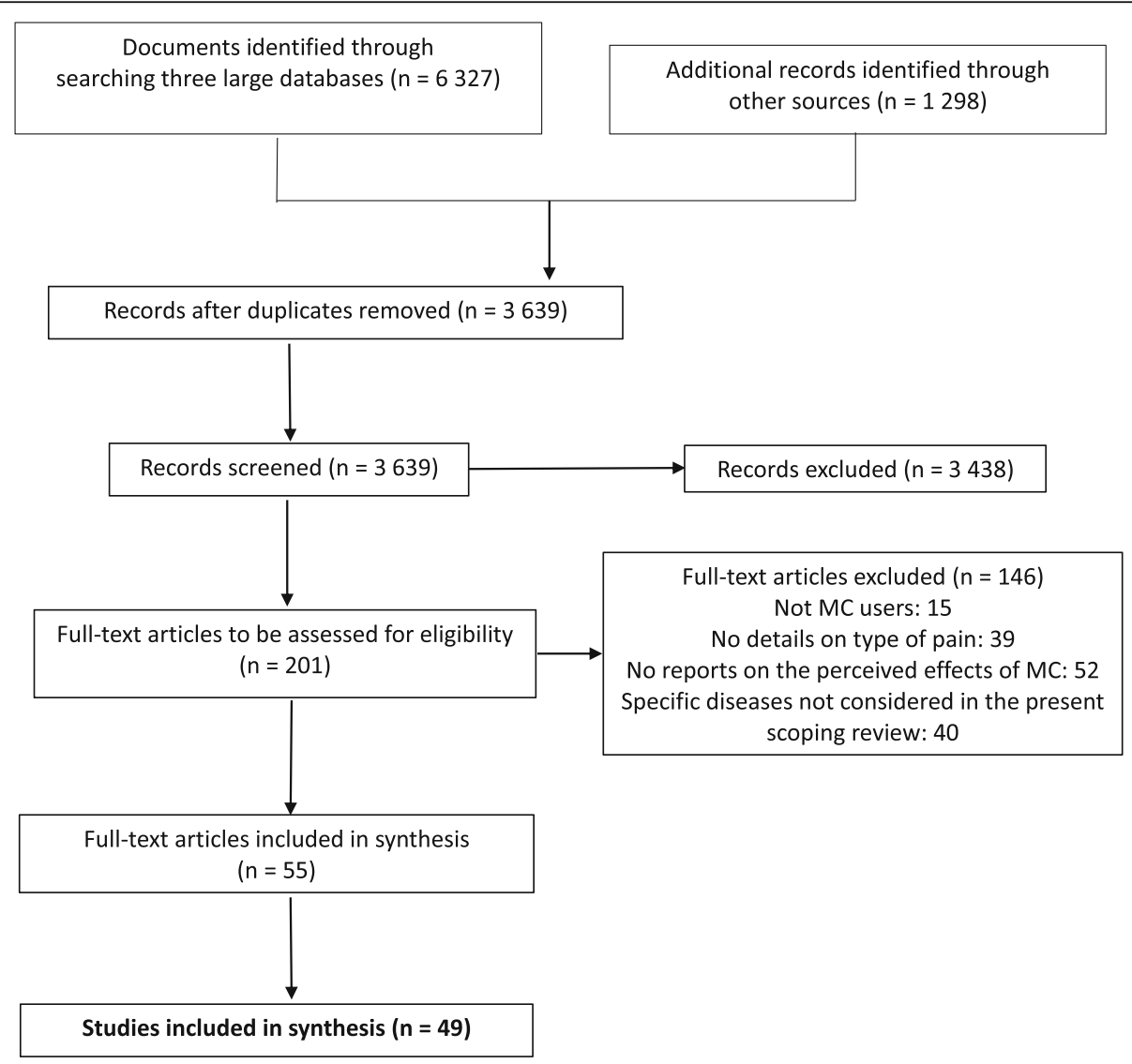

Fig. 1 Flowchart of the scoping review 


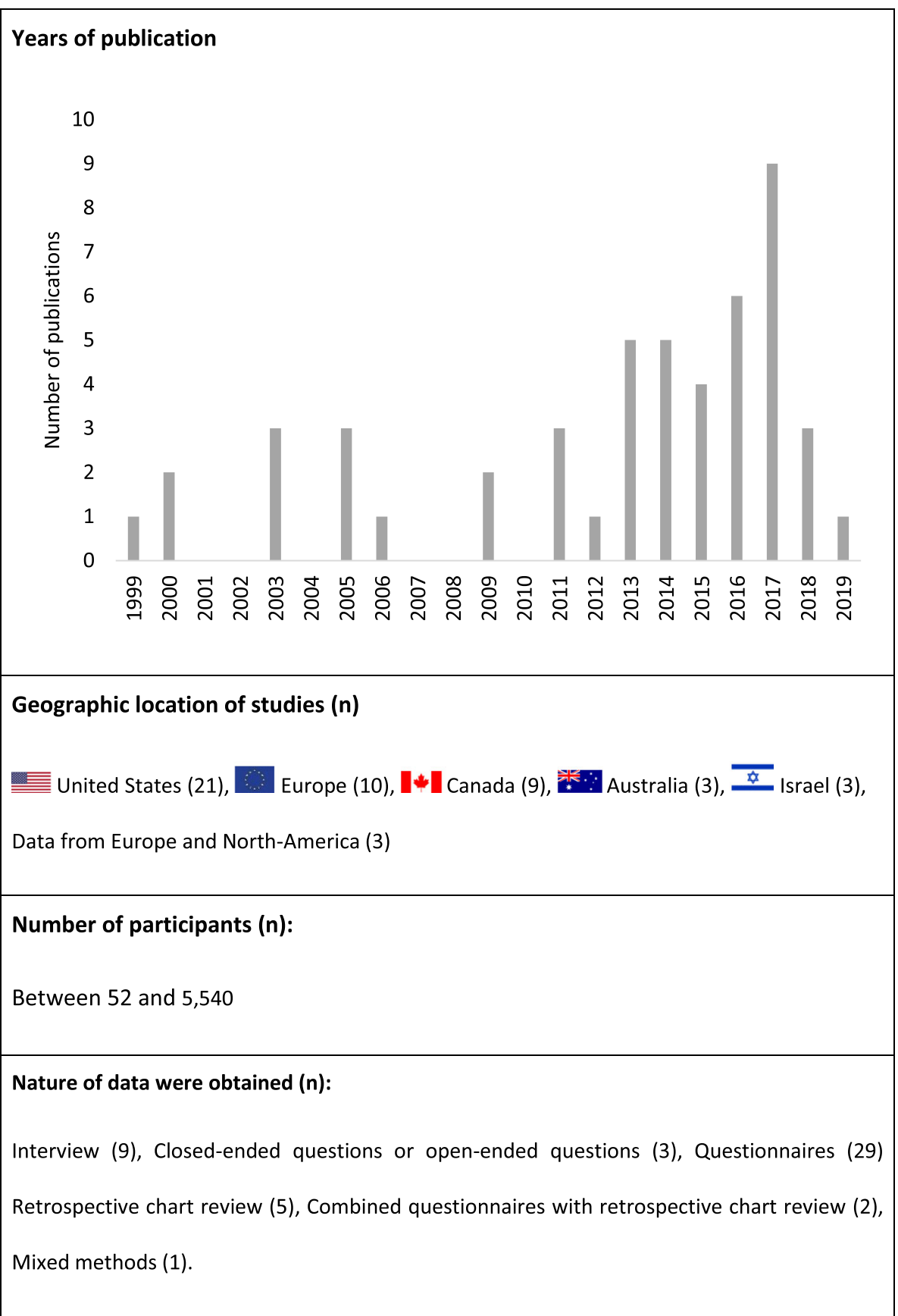

Fig. 2 Medical cannabis and musculoskeletal pain: scoping review key data

2013; Reiman, 2009; Reiman et al., 2017; Shah et al., 2017; Webb \& Webb, 2014; Zaller et al., 2015).

\section{Funding}

Funding information was reported in 28 of the 49 (57\%) studies (Table 1); 23 studies were funded by research grants or governmental scholarships (Aggarwal et al., 2009; Perron et al., 2015; Ste-Marie et al., 2016; Aggarwal et al., 2013a; Aggarwal et al., 2013b; Belle-Isle et al.,
2014; Bruce et al., 2018; Degenhardt et al., 2015; Erkens et al., 2005; Haroutounian et al., 2016; Harris et al., 2000; Hoffman et al., 2017; Lucas \& Walsh, 2017; Pedersen \& Sandberg, 2013; Sexton et al., 2016; Shiplo et al., 2016; Ste-Marie et al., 2012; Walsh et al., 2013; Brunt et al., 2014; Corroon Jr. et al., 2017; Cranford et al., 2016; Grella et al., 2014; Lavie-Ajayi \& Shvartzman, 2018). Two studies were supported by nongovernmental organizations (Hazekamp et al., 2013; 
Table 1 Brief summary of included studies

\begin{tabular}{|c|c|c|c|c|c|c|}
\hline \multirow[t]{2}{*}{ Article } & \multicolumn{2}{|l|}{ Study } & \multirow{2}{*}{$\begin{array}{l}\text { Participants } \\
\text { Number/age/sex }\end{array}$} & \multirow{2}{*}{$\begin{array}{l}\text { Reasons for using } \\
\text { cannabis medically }\end{array}$} & \multirow{2}{*}{$\begin{array}{l}\text { Reported effects and } \\
\text { perceptions of } \\
\text { medical cannabis }\end{array}$} & \multirow[t]{2}{*}{ Funding } \\
\hline & $\begin{array}{l}\text { Objectives/design: } \\
\text { data source; } \\
\text { recruitment }\end{array}$ & $\begin{array}{l}\text { Location } \\
\text { /period, legality }{ }^{1}\end{array}$ & & & & \\
\hline $\begin{array}{l}\text { Aggarwal } \\
\text { et al. } 2009\end{array}$ & $\begin{array}{l}\text { To characterize chronic } \\
\text { pain patients seeking } \\
\text { medical cannabis } \\
\text { treatment. } \\
\text { Quantitative: } \\
\text { Retrospective chart } \\
\text { review; recruitment via } \\
\text { a regional pain clinic. }\end{array}$ & $\begin{array}{l}\text { Washington State, } \\
\text { USA. } \\
\text { 2007-2008, study, } \\
\text { access points for } \\
\text { medical cannabis } \\
\text { dispensing in } \\
\text { urban centers } \\
\text { were informally } \\
\text { tolerated. }\end{array}$ & $\begin{array}{l}139 \text { patients seeking } \\
\text { treatment with } \\
\text { medical cannabis. } \\
\text { Median } 47 \text { (18-84) } \\
\text { years. } \\
63 \% \text { men. }\end{array}$ & $\begin{array}{l}\text { Chronic pain: } \\
82 \% \text { myofascial pain } \\
\text { syndrome } \\
64 \% \text { neuropathic pain } \\
27 \% \text { osteoarthritis. }\end{array}$ & $\begin{array}{l}\text { The majority of patient } \\
\text { records documented } \\
\text { significant symptom } \\
\text { alleviation. }\end{array}$ & $\begin{array}{l}\text { Scholarship } \\
\text { funding } \\
\text { *National Institute } \\
\text { of General Medical } \\
\text { Sciences of the NIH } \\
\text { "National Science } \\
\text { Foundation }\end{array}$ \\
\hline $\begin{array}{l}\text { Aggarwal } \\
\text { et al. (2013a \& } \\
\text { 2013b) }\end{array}$ & $\begin{array}{l}\text { To present data from a } \\
\text { dispensary-based survey } \\
\text { of medical cannabis } \\
\text { users. } \\
\text { Quantitative: } \\
\text { Dispensary-based sur- } \\
\text { vey; recruitment } \\
\text { through an medical } \\
\text { cannabis dispensary. }\end{array}$ & $\begin{array}{l}\text { Washington State, } \\
\text { USA. } \\
\text { 2007-2008, access } \\
\text { points for medical } \\
\text { cannabis } \\
\text { dispensing in } \\
\text { urban centers } \\
\text { were informally } \\
\text { tolerated. }\end{array}$ & $\begin{array}{l}37 \text { chronically ill, } \\
\text { qualified medical } \\
\text { cannabis users. } \\
41 \text { (21-61) years. } \\
65 \% \text { men. }\end{array}$ & $\begin{array}{l}25 \% \text { qualified with } \\
\text { intractable pain. } \\
51 \% \text { used medical } \\
\text { cannabis to reduce } \\
\text { musculoskeletal pain. }\end{array}$ & $\begin{array}{l}59 \% \text { of the participants } \\
\text { reported that } 3.4 \text { grams } \\
\text { of medical cannabis } \\
\text { provided } 97 \% \text { pain } \\
\text { relief for } 65 \mathrm{~h} \text {. }\end{array}$ & $\begin{array}{l}\text { Scholarship } \\
\text { funding } \\
\text { National Science } \\
\text { Foundation } \\
\text { Graduate Research } \\
\text { Fellowship }\end{array}$ \\
\hline $\begin{array}{l}\text { Alexandre } \\
2011\end{array}$ & $\begin{array}{l}\text { To identify patient's } \\
\text { expectations and } \\
\text { experience of the } \\
\text { enrollment to the } \\
\text { Rhode Island medical } \\
\text { cannabis program. } \\
\text { Qualitative: Semi- } \\
\text { structured face-to-face } \\
\text { interviews of patients } \\
\text { enrolled in the medical } \\
\text { cannabis program; re- } \\
\text { cruitment via an infor- } \\
\text { mation sheet } \\
\text { distributed by the } \\
\text { Rhode Island Patient } \\
\text { Advocacy Coalition } \\
\text { (RIPAC), supporting pa- } \\
\text { tients in the use of } \\
\text { medical cannabis. }\end{array}$ & $\begin{array}{l}\text { Rhode Island, } \\
\text { USA. } \\
\text { 2009-2010, legal } \\
\text { MC use. }\end{array}$ & $\begin{array}{l}15 \text { medical cannabis } \\
\text { qualified users enrolled } \\
\text { in the medical } \\
\text { cannabis program. } \\
23-60 \text { years. } \\
67 \% \text { men. }\end{array}$ & $\begin{array}{l}\text { Not reported for the } \\
\text { study sample (67\% of } \\
\text { registered users } \\
\text { diagnosed with chronic } \\
\text { or debilitating disease } \\
\text { or treatment, including } \\
\text { chronic pain not related } \\
\text { to cancer). }\end{array}$ & $\begin{array}{l}\text { Reports of significant } \\
\text { relief from pain. }\end{array}$ & No funding \\
\hline $\begin{array}{l}\text { Boehnke et al. } \\
2016\end{array}$ & $\begin{array}{l}\text { To examine whether } \\
\text { using medical cannabis } \\
\text { for chronic pain } \\
\text { changed individual } \\
\text { patterns of opioid use. } \\
\text { Quantitative: } \\
\text { Retrospective cross- } \\
\text { sectional survey (online } \\
\text { questionnaire carried } \\
\text { out in collaboration } \\
\text { with an medical canna- } \\
\text { bis dispensary) }\end{array}$ & $\begin{array}{l}\text { Michigan, USA. } \\
\text { 2013-2015 } \\
\text { Legal MC use. }\end{array}$ & $\begin{array}{l}185 \text { qualified medical } \\
\text { cannabis users who } \\
\text { completed the } 2011 \\
\text { Fibromyalgia Survey } \\
\text { Criteria. } \\
18-75 \text { years. } \\
64 \% \text { men. }\end{array}$ & Chronic pain. & $\begin{array}{l}\text { Medical cannabis use } \\
\text { was associated with a } \\
64 \% \text { decrease in opioid } \\
\text { use, decreased number } \\
\text { and side effects of } \\
\text { medications, and an } \\
\text { improved quality of life } \\
(45 \%) \text {. }\end{array}$ & N/A \\
\hline $\begin{array}{l}\text { Bonn-Miller } \\
\text { et al. } 2014\end{array}$ & $\begin{array}{l}\text { To describe population. } \\
\text { To examine association } \\
\text { psychological \& pain } \\
\text { symptoms vs. medical } \\
\text { cannabis use motives. } \\
\text { Quantitative: Cross- } \\
\text { sectional questionnaires; } \\
\text { recruitment via an med- } \\
\text { ical cannabis } \\
\text { dispensary. }\end{array}$ & $\begin{array}{l}\text { California, USA. } \\
\text { Legal medical } \\
\text { cannabis use. }\end{array}$ & $\begin{array}{l}217 \text { qualified medical } \\
\text { cannabis users } \\
\text { receiving medical } \\
\text { cannabis at dispensary. } \\
41.2 \pm 14.9 \text { years. } \\
73 \% \text { men. }\end{array}$ & $\begin{array}{l}62 \% \text { reported anxiety, } \\
58 \% \text { chronic pain, } 49 \% \\
\text { stress, } 48 \% \text { insomnia, } \\
45 \% \text { depression, } 30 \% \\
\text { appetite, } 26 \% \\
\text { headaches, } 22 \% \text { nausea, } \\
20 \% \text { muscle spasms, } \\
19 \% \text { PTSD; less than } \\
10 \% \text { of the sample } \\
\text { reported to use MC } \\
\text { against cancer. }\end{array}$ & $\begin{array}{l}\text { Regardless of condition, } \\
\text { medical cannabis } \\
\text { reported as moderately } \\
\text { to mostly helpful. }\end{array}$ & $\begin{array}{l}\text { (Mixed) } \\
\text { Research grant } \\
\text { VA Clinical Science } \\
\text { Research and } \\
\text { Development } \\
\text { (CSR\&D) Career } \\
\text { Development } \\
\text { Award-2 } \\
\text { Local resource } \\
\text { funding } \\
\text { San Francisco } \\
\text { Patient and } \\
\text { Resource Center }\end{array}$ \\
\hline $\begin{array}{l}\text { Bottorff et al. } \\
2011\end{array}$ & $\begin{array}{l}\text { To describe perceived } \\
\text { medical cannabis health } \\
\text { effects. } \\
\text { Qualitative: Semi- }\end{array}$ & $\begin{array}{l}\text { British Columbia, } \\
\text { Canada. } \\
\text { 2007-2008, } \\
\text { Marihuana }\end{array}$ & $\begin{array}{l}23 \text { self-reporting med- } \\
\text { ical cannabis users. } \\
45(25-66) \text { years. } \\
43 \% \text { men. }\end{array}$ & $\begin{array}{l}26 \% \text { HIV/AIDS } \\
22 \% \text { fibromyalgia } \\
17 \% \text { arthritis } \\
13 \% \text { mood/anxiety }\end{array}$ & $\begin{array}{l}\text { Reports of immediate } \\
\text { effects and, for the first } \\
\text { time in many years, } \\
\text { participants "could }\end{array}$ & N/A \\
\hline
\end{tabular}


Table 1 Brief summary of included studies (Continued)

\begin{tabular}{|c|c|c|c|c|c|c|}
\hline \multirow[t]{2}{*}{ Article } & \multicolumn{2}{|l|}{ Study } & \multirow{2}{*}{$\begin{array}{l}\text { Participants } \\
\text { Number/age/sex }\end{array}$} & \multirow{2}{*}{$\begin{array}{l}\text { Reasons for using } \\
\text { cannabis medically }\end{array}$} & \multirow{2}{*}{$\begin{array}{l}\text { Reported effects and } \\
\text { perceptions of } \\
\text { medical cannabis }\end{array}$} & \multirow[t]{2}{*}{ Funding } \\
\hline & $\begin{array}{l}\text { Objectives/design: } \\
\text { data source; } \\
\text { recruitment }\end{array}$ & $\begin{array}{l}\text { Location } \\
\text { /period, legality }\end{array}$ & & & & \\
\hline & $\begin{array}{l}\text { structured, individual } \\
\text { face-to-face or tele- } \\
\text { phone interviews; re- } \\
\text { cruitment through an } \\
\text { online forum and } \\
\text { through compassion } \\
\text { centers. }\end{array}$ & $\begin{array}{l}\text { Medical Access } \\
\text { Regulations * but } \\
\text { adults recruited } \\
\text { from tolerated } \\
\text { but illegal } \\
\text { dispensaries. }\end{array}$ & & disorders. & manage life again." & \\
\hline $\begin{array}{l}\text { Bruce et al. } \\
2018\end{array}$ & $\begin{array}{l}\text { To learn more on how } \\
\text { medical cannabis is } \\
\text { used by persons living } \\
\text { with chronic conditions } \\
\text { in tandem with or } \\
\text { instead of prescription } \\
\text { medications. } \\
\text { Qualitative: Semi- } \\
\text { structured telephone in- } \\
\text { terviews with open- } \\
\text { ended questions; re- } \\
\text { cruitment through flyers } \\
\text { at medical cannabis } \\
\text { dispensaries. }\end{array}$ & $\begin{array}{l}\text { Illinois, USA. } \\
\text { Legal medical } \\
\text { cannabis use. }\end{array}$ & $\begin{array}{l}30 \text { qualified medical } \\
\text { cannabis users. } \\
44.6 \pm 15.9 \text { years. } \\
63 \% \text { men. }\end{array}$ & $\begin{array}{l}23 \% \text { rheumatoid } \\
\text { arthritis } \\
20 \% \text { Crohn's disease } \\
20 \% \text { spinal cord injury/ } \\
\text { disease } \\
13 \% \text { cancer } \\
10 \% \text { severe } \\
\text { fibromyalgia. }\end{array}$ & $\begin{array}{l}\text { Medical cannabis } \\
\text { perceived as acting } \\
\text { more quickly, having } \\
\text { longer effects, reducing } \\
\text { potential harm versus } \\
\text { opioids/narcotics. } \\
\text { Multiple benefits } \\
\text { replacing a range of } \\
\text { medications. }\end{array}$ & $\begin{array}{l}\text { Fellowship grant } \\
\text { Provost's } \\
\text { Collaborative } \\
\text { Research } \\
\text { Fellowship, DePaul } \\
\text { University }\end{array}$ \\
\hline $\begin{array}{l}\text { Brunt et al. } \\
2014\end{array}$ & $\begin{array}{l}\text { To assess therapeutic } \\
\text { satisfaction with } \\
\text { pharmaceutical-grade } \\
\text { cannabis. } \\
\text { To compare the } \\
\text { subjective effects } \\
\text { among the available } \\
\text { strains. } \\
\text { Quantitative: } \\
\text { Questionnaires; } \\
\text { recruitment through } \\
\text { pharmacies specialized } \\
\text { in medical cannabis } \\
\text { distribution. }\end{array}$ & $\begin{array}{l}\text { The Netherlands. } \\
2011-2012, \\
\text { pharmaceutical- } \\
\text { grade cannabis } \\
\text { distributed for } \\
\text { medicinal pur- } \\
\text { poses since } 2003 \text {. }\end{array}$ & $\begin{array}{l}113 \text { qualified medical } \\
\text { cannabis users. } \\
52.8 \pm 12.3 \text { years. } \\
49 \% \text { men. }\end{array}$ & $\begin{array}{l}53 \% \text { chronic pain } \\
23 \% \text { multiple sclerosis; } \\
\text { only } 11 \% \text { indicated to } \\
\text { use medical cannabis } \\
\text { against cancer. }\end{array}$ & $\begin{array}{l}86 \% \text { (almost) always } \\
\text { experienced therapeutic } \\
\text { satisfaction, mainly pain } \\
\text { alleviation. }\end{array}$ & $\begin{array}{l}\text { Governmental } \\
\text { funding } \\
\text { Ministry of Health, } \\
\text { Welfare and Sport }\end{array}$ \\
\hline $\begin{array}{l}\text { Coomber } \\
\text { et al. } 2003\end{array}$ & $\begin{array}{l}\text { To report the } \\
\text { experiences of medical } \\
\text { cannabis users. } \\
\text { Qualitative: Semi } \\
\text { structured interviews; } \\
\text { recruitment via } \\
\text { advertisements in } \\
\text { newspapers, disabled } \\
\text { people's organizations } \\
\text { or friends. }\end{array}$ & $\begin{array}{l}\text { UK. } \\
\text { Illegal. }\end{array}$ & $\begin{array}{l}33 \text { self-identified med- } \\
\text { ical cannabis users. } \\
44 \text { (26-65) years. } \\
58 \% \text { men. }\end{array}$ & $\begin{array}{l}\text { To relieve symptoms of } \\
\text { chronic illness or } \\
\text { disability: } \\
42 \% \text { multiple sclerosis } \\
27 \% \text { arthritic/ } \\
\text { rheumatoid complaints. }\end{array}$ & $\begin{array}{l}\text { Medical cannabis } \\
\text { perceived to be highly } \\
\text { effective in treating } \\
\text { symptoms, to } \\
\text { complement existing } \\
\text { medication, and to } \\
\text { produce fewer } \\
\text { unwanted effects. }\end{array}$ & N/A \\
\hline $\begin{array}{l}\text { Corroon et al. } \\
2017\end{array}$ & $\begin{array}{l}\text { To survey cannabis users } \\
\text { to determine whether } \\
\text { they had intentionally } \\
\text { substituted cannabis for } \\
\text { prescription drugs. } \\
\text { Online survey, } \\
\text { recruitment through } \\
\text { social media, cannabis } \\
\text { dispensaries and word } \\
\text { of mouth. }\end{array}$ & $\begin{array}{l}83 \% \text { of the USA } \\
\text { (all } 50 \text { states } \\
\text { represented) and } \\
\text { over } 42 \text { countries } \\
\text { represented. } \\
2013-2016 \\
\text { Legality differed } \\
\text { between the USA } \\
\text { and countries. }\end{array}$ & $\begin{array}{l}\text { Convenience sample } \\
\text { of } 2774 \text { cannabis } \\
\text { users. } \\
63 \% \text { were under } 36 \text { y, } \\
56 \% \text { men. } \\
60 \% \text { identified } \\
\text { themselves as medical } \\
\text { cannabis users. }\end{array}$ & $\begin{array}{l}\text { 1040/2774 (37\%) of } \\
\text { respondents reported } \\
\text { pain and/or intractable } \\
\text { pain. }\end{array}$ & $\begin{array}{l}46 \% \text { have substituted } \\
\text { cannabis for } \\
\text { prescription drugs. }\end{array}$ & $\begin{array}{l}\text { Research grant } \\
\text { NIH NCCAM } \\
\text { K01ATTA (Ste-Marie } \\
\text { et al., 2016) }\end{array}$ \\
\hline $\begin{array}{l}\text { Cranford et al. } \\
2016\end{array}$ & $\begin{array}{l}\text { To examine the } \\
\text { prevalence and correlates } \\
\text { of vaporization as a route } \\
\text { of cannabis } \\
\text { administration in medical }\end{array}$ & $\begin{array}{l}\text { Michigan, USA. } \\
\text { 2014-2015 } \\
\text { Legal medical } \\
\text { cannabis use. }\end{array}$ & $\begin{array}{l}1485 \text { adults seeking } \\
\text { medical cannabis } \\
\text { certification either for } \\
\text { the first time or as a } \\
\text { renewal }(66 \%) \text {. }\end{array}$ & $\begin{array}{l}91 \% \text { severe chronic } \\
\text { pain } \\
26 \% \text { severe and } \\
\text { persistent muscle } \\
\text { spasms. }\end{array}$ & not reported & $\begin{array}{l}\text { Research grant } \\
\text { National Institute } \\
\text { on Drug Abuse } \\
\text { (NIDA), National } \\
\text { Institutes of Health }\end{array}$ \\
\hline
\end{tabular}

Quantitative: Data from the screening assessment; recruitment at medical cannabis clinics. 
Table 1 Brief summary of included studies (Continued)

\begin{tabular}{|c|c|c|c|c|c|c|}
\hline \multirow[t]{2}{*}{ Article } & \multicolumn{2}{|l|}{ Study } & \multirow{2}{*}{$\begin{array}{l}\text { Participants } \\
\text { Number/age/sex }\end{array}$} & \multirow{2}{*}{$\begin{array}{l}\text { Reasons for using } \\
\text { cannabis medically }\end{array}$} & \multirow{2}{*}{$\begin{array}{l}\text { Reported effects and } \\
\text { perceptions of } \\
\text { medical cannabis }\end{array}$} & \multirow[t]{2}{*}{ Funding } \\
\hline & $\begin{array}{l}\text { Objectives/design: } \\
\text { data source; } \\
\text { recruitment }\end{array}$ & $\begin{array}{l}\text { Location } \\
\text { /period, legality }{ }^{1}\end{array}$ & & & & \\
\hline Crowell 2017 & $\begin{array}{l}\text { To ascertain the impact } \\
\text { of medical cannabis on } \\
\text { patients in New Jersey. } \\
\text { Quantitative: Survey } \\
\text { with open-ended ques- } \\
\text { tions; recruitment via a } \\
\text { non-profit organization } \\
\text { dispensing medical } \\
\text { cannabis }\end{array}$ & $\begin{array}{l}\text { New Jersey, USA. } \\
\text { Legal medical } \\
\text { cannabis use. }\end{array}$ & $\begin{array}{l}955 \text { qualified medical } \\
\text { cannabis users. } \\
49.3 \pm 13.6(9-84) \\
\text { years. } \\
51 \% \text { men. }\end{array}$ & $\begin{array}{l}17 \text { conditions were } \\
\text { listed, including: } \\
28 \% \text { intractable skeletal } \\
\text { spasticity } \\
24 \% \text { chronic/severe } \\
\text { pain } \\
16 \% \text { multiple sclerosis } \\
11 \% \text { inflammatory } \\
\text { bowel disease. }\end{array}$ & $\begin{array}{l}\text { Improvement to } \\
\text { general condition and } \\
\text { quality of life. Decrease } \\
\text { in pain, inflammation, } \\
\text { nausea, intraocular } \\
\text { pressure, spasms, } \\
\text { seizure. Increase in } \\
\text { appetite, mobility, } \\
\text { mood and energy. }\end{array}$ & N/A \\
\hline $\begin{array}{l}\text { Degenhardt } \\
\text { et al. } 2015\end{array}$ & $\begin{array}{l}\text { To investigate patterns } \\
\text { and correlates of } \\
\text { cannabis use in people } \\
\text { who had been } \\
\text { prescribed opioids for } \\
\text { chronic non-cancer } \\
\text { pain. } \\
\text { Qualitative: Interview; } \\
\text { recruitment via a } \\
\text { database of pharmacies } \\
\text { and chemists across } \\
\text { Australia. }\end{array}$ & $\begin{array}{l}\text { Australia. } \\
\text { Legal medical } \\
\text { cannabis use. }\end{array}$ & $\begin{array}{l}242 \text { patients } \\
\text { prescribed opioids for } \\
\text { chronic non-cancer } \\
\text { pain which had used } \\
\text { cannabis for pain. } \\
48.7 \pm 10.1 \text { years. } \\
62.5 \% \text { men. }\end{array}$ & $\begin{array}{l}\text { Chronic non-cancer } \\
\text { pain, including: } \\
84 \% \text { back/neck } \\
\text { problems } \\
57 \% \text { arthritis/ } \\
\text { rheumatism. }\end{array}$ & $\begin{array}{l}\text { Among those using } \\
\text { cannabis for pain, the } \\
\text { average pain relief was } \\
70 \% \text { while the average } \\
\text { pain relief from } \\
\text { prescribed opioids was } \\
50 \% \text {. }\end{array}$ & $\begin{array}{l}\text { Research grant } \\
\text { Australian National } \\
\text { Health and } \\
\text { Medical Research } \\
\text { Council }\end{array}$ \\
\hline $\begin{array}{l}\text { Erkens et al. } \\
2005\end{array}$ & $\begin{array}{l}\text { To characterize medical } \\
\text { cannabis users, } \\
\text { symptoms and } \\
\text { conditions; daily use of } \\
\text { medical cannabis. } \\
\text { Quantitative: Structured } \\
\text { questionnaire; } \\
\text { recruitment via } \\
\text { pharmacies. }\end{array}$ & $\begin{array}{l}\text { Netherlands. } \\
\text { 2003-2004, since } \\
\text { 2003, } \\
\text { pharmaceutical- } \\
\text { grade cannabis is } \\
\text { distributed for } \\
\text { medicinal } \\
\text { purposes. }\end{array}$ & $\begin{array}{l}200 \text { patients who filled } \\
\text { a prescription for } \\
\text { medical cannabis. } \\
\geq 30 \text { years. } \\
33 \% \text { men. }\end{array}$ & $\begin{array}{l}\text { Cannabis mainly used } \\
\text { for chronic pain } \\
\text { (including rheumatic } \\
\text { disease) and muscle } \\
\text { cramp/stiffness. }\end{array}$ & Not reported & $\begin{array}{l}\text { Governmental } \\
\text { funding } \\
\text { Ministry of Health, } \\
\text { Welfare and } \\
\text { Sports, The } \\
\text { Netherlands }\end{array}$ \\
\hline $\begin{array}{l}\text { Fanelli et al. } \\
2017\end{array}$ & $\begin{array}{l}\text { To present the first } \\
\text { snapshot of the Italian } \\
\text { experience with } \\
\text { cannabis use for } \\
\text { chronic pain over the } \\
\text { initial year of its use. } \\
\text { Quantitative: } \\
\text { Retrospective case } \\
\text { series (physician-filled } \\
\text { case report form); } \\
\text { recruitment via second- } \\
\text { level pain clinics. }\end{array}$ & $\begin{array}{l}\text { Pisa, Italy. } \\
2015-2016 \text {, initial } \\
\text { year of authorized } \\
\text { medical cannabis } \\
\text { use for chronic } \\
\text { pain. } \\
\text { Legal medical } \\
\text { cannabis use. }\end{array}$ & $\begin{array}{l}614 \text { qualified medical } \\
\text { cannabis users. } \\
61.3 \pm 15.3 \text { years. } \\
29 \% \text { men. }\end{array}$ & 91\% chronic pain. & $\begin{array}{l}49 \% \text { reported an } \\
\text { improvement } \\
\text { associated with the } \\
\text { therapy. } \\
15 \% \text { stopped the } \\
\text { treatment due to side } \\
\text { effects (none of which } \\
\text { were severe). }\end{array}$ & N/A \\
\hline $\begin{array}{l}\text { Gorter et al. } \\
2005\end{array}$ & $\begin{array}{l}\text { To investigate } \\
\text { indications for cannabis } \\
\text { prescription. } \\
\text { To assess cannabis } \\
\text { efficacy and side effects. } \\
\text { Quantitative: } \\
\text { Standardized } \\
\text { questionnaire; } \\
\text { recruitment via } \\
\text { questionnaires } \\
\text { accompanying } \\
\text { shipment of medical- } \\
\text { grade cannabis directed } \\
\text { to both patient and } \\
\text { prescribing physician. }\end{array}$ & $\begin{array}{l}\text { Netherlands. } \\
\text { 1997-1999, before } \\
\text { legalization but } \\
\text { consumption of } \\
\text { small amounts } \\
\text { under certain } \\
\text { conditions was } \\
\text { then condoned. }\end{array}$ & $\begin{array}{l}107 \text { patients receiving } \\
\text { medical-grade canna- } \\
\text { bis on prescription. } \\
\text { Median } 58 \text { years. } \\
45 \% \text { men. }\end{array}$ & $\begin{array}{l}39 \% \text { neurologic } \\
\text { disorders } \\
21 \% \text { musculoskeletal/ } \\
\text { connective tissue } \\
\text { disorders } \\
14 \% \text { malignant tumors } \\
\text { and symptoms thereof. }\end{array}$ & $\begin{array}{l}64 \% \text { reported good to } \\
\text { excellent effect on their } \\
\text { symptoms. } \\
\text { Generally mild side } \\
\text { effects. }\end{array}$ & $\begin{array}{l}\text { Non- } \\
\text { governmental } \\
\text { organization } \\
\text { funding } \\
\text { Maripharm } \\
\end{array}$ \\
\hline $\begin{array}{l}\text { Grella et al. } \\
2014\end{array}$ & $\begin{array}{l}\text { To collect descriptive } \\
\text { data on individuals } \\
\text { using medical cannabis } \\
\text { dispensaries. } \\
\text { Mixed } \\
\text { Focus groups and } \\
\text { survey; recruitment via } \\
\text { medical cannabis } \\
\text { dispensaries. } S\end{array}$ & $\begin{array}{l}\text { California, USA. } \\
\text { May-October } \\
\text { 2012, legal } \\
\text { medical cannabis } \\
\text { use. }\end{array}$ & $\begin{array}{l}\text { Users of medical } \\
\text { cannabis dispensaries: } \\
\text { Focus groups: } n=30 \\
38 \pm 12(20-64) \text { y, } 70 \% \\
\text { men. } \\
\text { Survey: } n=182,28.4 \pm \\
5.3 \text { y, } 74 \% \text { men. }\end{array}$ & $\begin{array}{l}\text { Conditions most often } \\
\text { cited (not mutually } \\
\text { exclusive): } \\
60 \% \text { anxiety } \\
56 \% \text { insomnia/sleep } \\
\text { problems } \\
33 \% \text { depression } \\
42 \% \text { chronic (non- } \\
\text { cancer) pain. }\end{array}$ & $\begin{array}{l}\text { Nearly all believed MC } \\
\text { beneficial in treating } \\
\text { their health problems. }\end{array}$ & $\begin{array}{l}\text { Governmental } \\
\text { funding } \\
\text { Los Angeles County } \\
\text { Department of } \\
\text { Public Health, } \\
\text { Substance Abuse } \\
\text { Prevention and } \\
\text { Control Programs }\end{array}$ \\
\hline
\end{tabular}


Table 1 Brief summary of included studies (Continued)

\begin{tabular}{|c|c|c|c|c|c|c|}
\hline \multirow[t]{2}{*}{ Article } & \multicolumn{2}{|l|}{ Study } & \multirow{2}{*}{$\begin{array}{l}\text { Participants } \\
\text { Number/age/sex }\end{array}$} & \multirow{2}{*}{$\begin{array}{l}\text { Reasons for using } \\
\text { cannabis medically }\end{array}$} & \multirow{2}{*}{$\begin{array}{l}\text { Reported effects and } \\
\text { perceptions of } \\
\text { medical cannabis }\end{array}$} & \multirow[t]{2}{*}{ Funding } \\
\hline & $\begin{array}{l}\text { Objectives/design: } \\
\text { data source; } \\
\text { recruitment }\end{array}$ & $\begin{array}{l}\text { Location } \\
\text { /period, legality }{ }^{1}\end{array}$ & & & & \\
\hline $\begin{array}{l}\text { Groten- } \\
\text { hermen \& } \\
\text { Schnelle } 2003\end{array}$ & $\begin{array}{l}\text { To investigate indications } \\
\text { for cannabis prescription. } \\
\text { To assess cannabis } \\
\text { efficacy and side effects. } \\
\text { Quantitative: } \\
\text { Questionnaires; } \\
\text { recruitment via an } \\
\text { medical cannabis } \\
\text { association. }\end{array}$ & $\begin{array}{l}\text { German speech } \\
\text { area of Europe. } \\
\text { 2001: illegal use } \\
\text { of natural } \\
\text { cannabis products } \\
\text { but THC could be } \\
\text { prescribed. }\end{array}$ & $\begin{array}{l}143 \text { participants with } \\
\text { cannabis or THC } \\
\text { experience. } \\
\text { Median } 40.3 \text { (16-87) } \\
\text { years. } \\
61 \% \text { men. }\end{array}$ & $\begin{array}{l}28 \% \text { neurological } \\
\text { symptoms } \\
25 \% \text { painful conditions. }\end{array}$ & $\begin{array}{l}75 \% \text { reported their } \\
\text { conditions much } \\
\text { improved by cannabis } \\
\text { or THC. } \\
73 \% \text { reported no side } \\
\text { effects. }\end{array}$ & $N \backslash A$ \\
\hline $\begin{array}{l}\text { Haroutounian } \\
\text { et al. } 2016\end{array}$ & $\begin{array}{l}\text { To determine the long- } \\
\text { term effect of medical } \\
\text { cannabis on pain and } \\
\text { functional outcomes in } \\
\text { participants with treat- } \\
\text { ment resistant chronic } \\
\text { pain. } \\
\text { Quantitative: } \\
\text { Prospective, open-label, } \\
\text { single-arm longitudinal } \\
\text { study (questionnaires); } \\
\text { recruitment via an am- } \\
\text { bulatory pain clinic. }\end{array}$ & $\begin{array}{l}\text { Jerusalem, Israel. } \\
\text { 2010-2013, legal } \\
\text { medical cannabis } \\
\text { use. }\end{array}$ & $\begin{array}{l}206 \text { qualified medical } \\
\text { cannabis users. } \\
51.2 \pm 15.4 \text { years } \\
62 \% \text { men. }\end{array}$ & $\begin{array}{l}93 \% \text { chronic non-cancer } \\
\text { pain, including: } \\
37 \% \text { musculoskeletal } \\
\text { pain } \\
34 \% \text { peripheral } \\
\text { neuropathic pain } \\
19 \% \text { radicular low back } \\
\text { pain. }\end{array}$ & $\begin{array}{l}\text { Pain symptom score } \\
\text { improved }(P<0.001) \text { in } \\
\text { association with } \\
\text { improvement in } \\
\text { physical function ( } P< \\
0.001) \text {. } \\
9 \text { (4\%) discontinued } \\
\text { treatment due to mild } \\
\text { to moderate AEs; } 2 \text { (1\%) } \\
\text { discontinued to serious } \\
\text { side effects (1 elevated } \\
\text { liver transaminases, } 1 \\
\text { elderly admitted to an } \\
\text { Emergency Department } \\
\text { in a confusional state). }\end{array}$ & $\begin{array}{l}\text { Research grant } \\
\text { Support from the } \\
\text { Hadassah-Hebrew } \\
\text { University Pain Re- } \\
\text { lief Unit }\end{array}$ \\
\hline $\begin{array}{l}\text { Harris et al. } \\
2000\end{array}$ & $\begin{array}{l}\text { To better understand } \\
\text { relationships between } \\
\text { past experience with } \\
\text { drugs and reasons for } \\
\text { cannabis use; perceived } \\
\text { effectiveness of cannabis } \\
\text { as a therapeutic agent. } \\
\text { Quantitative: } \\
\text { Questionnaires; } \\
\text { recruitment via } \\
\text { advertisements posted } \\
\text { at the Cannabis } \\
\text { Cultivator's Club. }\end{array}$ & $\begin{array}{l}\text { California, USA } \\
\text { (after 1996) } \\
\text { Legal MC use. }\end{array}$ & $\begin{array}{l}100 \text { Cannabis } \\
\text { Cultivator's Club } \\
\text { members. } \\
40 \pm 8 \text { years. } \\
78 \% \text { men. }\end{array}$ & $\begin{array}{l}33 \% \text { AIDS (appetite) } \\
21 \% \text { musculoskeletal/ } \\
\text { arthritis } \\
15 \% \text { gastrointestinal } \\
\text { (most often nausea) } \\
15 \% \text { psychiatric } \\
\text { (primarily depression) } \\
13 \% \text { neurologic and } \\
\text { non-musculoskeletal } \\
\text { pain syndromes. }\end{array}$ & $\begin{array}{l}66 \% \text { rated effectiveness } \\
\text { as } 80 \% \text { compared with } \\
52 \% \text { for other } \\
\text { medications. } \\
56 \% \text { reported no side } \\
\text { effects. } \\
\text { Less severe side effects } \\
\text { than other treatments. } \\
\text { Anxiety effects } \\
\text { frequently reported on } \\
\text { the checklist but not } \\
\text { listed as side effects. }\end{array}$ & $\begin{array}{l}\text { Research grant } \\
\text { US Public Health } \\
\text { Service grants, } \\
\text { National Institute } \\
\text { on Drug Abuse }\end{array}$ \\
\hline $\begin{array}{l}\text { Hazekamp \& } \\
\text { Heerdink, } \\
2013\end{array}$ & $\begin{array}{l}\text { To analyze the } \\
\text { incidence and } \\
\text { prevalence of medical } \\
\text { cannabis use and } \\
\text { characteristics of users. } \\
\text { Quantitative: } \\
\text { Retrospective database } \\
\text { study; recruitment } \\
\text { through the Dutch } \\
\text { Foundation for } \\
\text { Pharmaceutical } \\
\text { Statistics and the only } \\
\text { Dutch pharmacy } \\
\text { specialized in medical } \\
\text { cannabis dispensing. }\end{array}$ & $\begin{array}{l}\text { Netherlands. } \\
\text { 2003-2010, } \\
\text { pharmaceutical- } \\
\text { grade cannabis } \\
\text { distributed for } \\
\text { medicinal pur- } \\
\text { poses since } 2003 \text {. }\end{array}$ & $\begin{array}{l}5540 \text { patients with } \geq 1 \\
\text { medical cannabis } \\
\text { prescription. } \\
56 \text { (14-93) years. } \\
43 \% \text { men. }\end{array}$ & $\begin{array}{l}\text { Reason for medical } \\
\text { cannabis use not } \\
\text { reported but } 43 \% \text { had } \\
\text { analgesics prescribed in } \\
\text { the } 6 \text {-month period } \\
\text { preceding start of MC } \\
\text { use. Only } 2.7 \% \text { received } \\
\text { oncologicals, thus can- } \\
\text { cer is unlikely to be } \\
\text { present in all pain pa- } \\
\text { tients in the study. }\end{array}$ & not reported & N/A \\
\hline $\begin{array}{l}\text { Hazekamp } \\
\text { et al. } 2013\end{array}$ & $\begin{array}{l}\text { To compare different } \\
\text { administration forms of } \\
\text { cannabinoids and } \\
\text { identify their relative } \\
\text { advantages and } \\
\text { disadvantages as } \\
\text { described by actual } \\
\text { users. } \\
\text { International, web- } \\
\text { based, cross-sectional }\end{array}$ & $\begin{array}{l}31 \text { countries } \\
\text { including the USA } \\
\text { (40 states } \\
\text { represented), } \\
\text { Germany, France, } \\
\text { Canada, } \\
\text { Netherlands \& } \\
\text { Spain. } \\
\text { 2009-2010, } \\
\text { legality differed }\end{array}$ & $\begin{array}{l}953 \text { adults self- } \\
\text { reporting experience } \\
\text { with } \geq 2 \text { different } \\
\text { cannabinoid-based } \\
\text { medicines or adminis- } \\
\text { tration forms, } 87 \% \\
\text { current medical canna- } \\
\text { bis users. } \\
40.7 \text { (14-76) years. } \\
64 \% \text { men. }\end{array}$ & $\begin{array}{l}\text { Top } 5 \text { conditions: } \\
12 \% \text { back pain } \\
7 \% \text { sleeping disorder } \\
7 \% \text { depression } \\
6 \% \text { pain resulting from } \\
\text { injury or accident } \\
4 \% \text { multiple sclerosis. } \\
\text { Pain medication was } \\
\text { consumed by } 53.6 \% \text { of } \\
\text { medical cannabis users }\end{array}$ & $\begin{array}{l}\text { Herbal medical } \\
\text { cannabis received } \\
\text { higher appreciation } \\
\text { than pharmaceutical } \\
\text { cannabinoids. } \\
\text { Side effects: irritation of } \\
\text { the lungs (inhalation), } \\
\text { drowsiness, } \\
\text { uncontrollable appetite, } \\
\text { "getting high". }\end{array}$ & $\begin{array}{l}\text { Non- } \\
\text { governmental } \\
\text { organization } \\
\text { funding } \\
\text { Dutch Association } \\
\text { for Legal Cannabis } \\
\text { and its } \\
\text { Constituents as } \\
\text { Medicine (NCSM } \\
\text { foundation) }\end{array}$ \\
\hline
\end{tabular}


Table 1 Brief summary of included studies (Continued)

\begin{tabular}{|c|c|c|c|c|c|c|}
\hline \multirow[t]{2}{*}{ Article } & \multicolumn{2}{|l|}{ Study } & \multirow{2}{*}{$\begin{array}{l}\text { Participants } \\
\text { Number/age/sex }\end{array}$} & \multirow{2}{*}{$\begin{array}{l}\text { Reasons for using } \\
\text { cannabis medically }\end{array}$} & \multirow{2}{*}{$\begin{array}{l}\text { Reported effects and } \\
\text { perceptions of } \\
\text { medical cannabis }\end{array}$} & \multirow[t]{2}{*}{ Funding } \\
\hline & $\begin{array}{l}\text { Objectives/design: } \\
\text { data source; } \\
\text { recruitment }\end{array}$ & $\begin{array}{l}\text { Location } \\
\text { /period, legality }{ }^{1}\end{array}$ & & & & \\
\hline $\begin{array}{l}\text { Hoffman et al. } \\
2017\end{array}$ & $\begin{array}{l}\text { To begin the } \\
\text { development of a } \\
\text { cannabis use registry in } \\
\text { Oregon. } \\
\text { Qualitative: Semi- } \\
\text { structured interviews; } \\
\text { recruitment via an out- } \\
\text { patient healthcare } \\
\text { clinic. }\end{array}$ & $\begin{array}{l}\text { Oregon, USA. } \\
\text { July-August 2015: } \\
\text { legal medical } \\
\text { cannabis use, } \\
\text { nonmedical used } \\
\text { became legal on } \\
\text { July first. }\end{array}$ & $\begin{array}{l}22 \text { qualified medical } \\
\text { cannabis users. } \\
\text { Median } 38 \text { (20-64) } \\
\text { years. } \\
45 \% \text { men. }\end{array}$ & $\begin{array}{l}59 \% \text { musculoskeletal } \\
\text { pain } \\
27 \% \text { PTSD. }\end{array}$ & $\begin{array}{l}\text { Some reported } \\
\text { physiologic relief from } \\
\text { pain, others said it } \\
\text { helped take their mind } \\
\text { off of it. } \\
\text { Respondents felt that } \\
\text { the benefits } \\
\text { outweighed the risks. }\end{array}$ & $\begin{array}{l}\text { Research grant } \\
\text { National Institute } \\
\text { of Drug Abuse } \\
\text { supported this } \\
\text { study }\end{array}$ \\
\hline $\begin{array}{l}\text { Ilgen et al. } \\
2013\end{array}$ & $\begin{array}{l}\text { To describe adults } \\
\text { seeking medical } \\
\text { cannabis; } \\
\text { To compare them with } \\
\text { those renewing their } \\
\text { medical cannabis card } \\
\text { on substance use; pain; } \\
\text { functioning. } \\
\text { Quantitative: } \\
\text { Questionnaires; } \\
\text { recruitment at the } \\
\text { waiting room of an } \\
\text { medical cannabis clinic. }\end{array}$ & $\begin{array}{l}\text { Michigan, USA. } \\
\text { Legal medical } \\
\text { cannabis use. }\end{array}$ & $\begin{array}{l}348 \text { adults seeking } \\
\text { medical cannabis } \\
\text { certification either for } \\
\text { the first time }(56 \%) \text { or } \\
\text { as a renewal }(44 \%) . \\
41.5 \pm 12.6 \text { years. } \\
66 \% \text { men. }\end{array}$ & $\begin{array}{l}87 \% \text { used medical } \\
\text { cannabis for pain relief, } \\
\text { including } 7 \% \text { for } \\
\text { musculoskeletal } \\
\text { problems. }\end{array}$ & Not reported & N/A \\
\hline $\begin{array}{l}\text { Kilcher et al. } \\
2017\end{array}$ & $\begin{array}{l}\text { To study medical uses } \\
\text { of cannabinoids as part } \\
\text { of the Swiss Federal } \\
\text { Office of Public Health } \\
\text { (FOPH) programme of } \\
\text { exceptional licenses. } \\
\text { Quantitative: Data from } \\
\text { the formal requests for } \\
\text { medical use of } \\
\text { cannabinoids; } \\
\text { recruitment via formal } \\
\text { requests of medical } \\
\text { cannabis use. }\end{array}$ & $\begin{array}{l}\text { Switzerland. } \\
\text { 2013-2014, } \\
\text { exceptional } \\
\text { licenses for } \\
\text { medical use of } \\
\text { cannabinoids. }\end{array}$ & $\begin{array}{l}1193 \text { qualified medical } \\
\text { cannabis users. } \\
57 \pm 15 \text { years. } \\
43 \% \text { men. }\end{array}$ & $\begin{array}{l}\text { Most common } \\
\text { symptoms:49\% chronic } \\
\text { pain40\% Spasticity } \\
\text { Diagnosis:25\% } \\
\text { musculoskeletal } \\
\text { conditions22\% multiple } \\
\text { sclerosis. }\end{array}$ & $\begin{array}{l}\text { Licences were initially } \\
\text { granted for } 6 \text { months, } \\
\text { physicians requested } \\
\text { extensions when the } \\
\text { treatment had been } \\
\text { satisfactory. The } \\
\text { number of extensions } \\
\text { increased from } 26 \% \text { in } \\
2013 \text { to } 39 \% \text { in } 2014 \text {. }\end{array}$ & N/A \\
\hline $\begin{array}{l}\text { Lavie-Ajayi \& } \\
\text { Shvartzman } \\
2018\end{array}$ & $\begin{array}{l}\text { To evaluate the } \\
\text { subjective experience of } \\
\text { pain relief by medical } \\
\text { cannabis. } \\
\text { Qualitative: In-depth } \\
\text { semistructured inter- } \\
\text { views; recruitment } \\
\text { through a pain clinic. }\end{array}$ & $\begin{array}{l}\text { Israel. } \\
\text { 2016-2017, legal } \\
\text { medical cannabis } \\
\text { use. }\end{array}$ & $\begin{array}{l}19 \text { patients seeking } \\
\text { treatment with } \\
\text { medical cannabis. } \\
52(28-79) \text { years. } \\
53 \% \text { men }\end{array}$ & $\begin{array}{l}\text { Chronic pain: } 37 \% \\
\text { arthritis32\% spinal cord } \\
\text { injuries } 32 \% \text { CRPS } \\
\text { 5\% cancer. }\end{array}$ & $\begin{array}{l}\text { Immediate sensation of } \\
\text { chronic pain relief, } \\
\text { improved sleep quality, } \\
\text { improved life quality. } \\
\text { Side effects: increased } \\
\text { appetite }(74 \%), \\
\text { drowsiness }(67.1 \%), \\
\text { ocular irritation (40.7\%), } \\
\text { lack of energy (37.5\%), } \\
\text { memory impairment } \\
(31.6 \%), \text { palpitations } \\
(15.4 \%), \text { and paranoia } \\
(15.2 \%) \text { or confusion } \\
(12.4 \%) .\end{array}$ & $\begin{array}{l}\text { Research grant } \\
\text { Ben Gurion } \\
\text { University of the } \\
\text { Negev, Faculty of } \\
\text { Humanities and } \\
\text { Social Sciences. }\end{array}$ \\
\hline $\begin{array}{l}\text { Lintzeris et al., } \\
2018\end{array}$ & $\begin{array}{l}\text { To explore patterns of } \\
\text { medical cannabis use. } \\
\text { Quantitative: Online } \\
\text { survey; recruitment } \\
\text { trough online media, } \\
\text { consumer group } \\
\text { webpages, and medical } \\
\text { cannabis consumer } \\
\text { forums. }\end{array}$ & $\begin{array}{l}\text { Australia. } \\
\text { 2016, illegal } \\
\text { medical cannabis } \\
\text { use. }\end{array}$ & $\begin{array}{l}1748 \text { medical cannabis } \\
\text { users. } \\
37.9 \text { years. } \\
68 \% \text { men. }\end{array}$ & $\begin{array}{l}51 \% \text { anxiety, } 50 \% \text { back } \\
\text { pain, } 49 \% \text { depression, } \\
44 \% \text { sleep problems, } \\
26 \% \text { neck pain, } 23 \% \\
\text { PTSD. } 69.4 \% \text { of } \\
\text { respondents used } \\
\text { medical cannabis to } \\
\text { manage pain. }\end{array}$ & $\begin{array}{l}\text { Most participants } \\
\text { reported that medical } \\
\text { cannabis reduced } \\
\text { significantly chronic } \\
\text { pain. } \\
\text { Side effects: increased } \\
\text { appetite }(74 \%), \\
\text { drowsiness }(67 \%), \\
\text { ocular irritation (41\%), } \\
\text { lack of energy (38\%), } \\
\text { memory impairment } \\
(32 \%), \text { palpitations } \\
(16 \%) \text {, paranoia (15\%) or } \\
\text { confusion (12\%). }\end{array}$ & $\begin{array}{l}\text { (Mixed) } \\
\text { Research grant } \\
\text { Australian } \\
\text { Research Council } \\
\text { and the National } \\
\text { Health and } \\
\text { Medical Research } \\
\text { council (NHMRC) } \\
\text { Local research } \\
\text { grant } \\
\text { Lambert Initiative } \\
\text { for Cannabinoid } \\
\text { Therapeutics }\end{array}$ \\
\hline $\begin{array}{l}\text { Lucas \& Walsh } \\
2017\end{array}$ & $\begin{array}{l}\text { To describe medical } \\
\text { cannabis access, use } \\
\text { and substitution for }\end{array}$ & $\begin{array}{l}\text { Canada. } \\
\text { July 2015, legal } \\
\text { medical cannabis }\end{array}$ & $\begin{array}{l}271 \text { qualified medical } \\
\text { cannabis users } \\
\text { (Marihuana for Medical }\end{array}$ & $\begin{array}{l}\text { 53\% pain-related } \\
\text { conditions: } \\
36 \% \text { chronic pain, } 12 \%\end{array}$ & $\begin{array}{l}95 \% \text { reported that } \\
\text { cannabis often or } \\
\text { always helped alleviate }\end{array}$ & $\begin{array}{l}\text { Research grant } \\
\text { Institute for } \\
\text { Healthy Living and }\end{array}$ \\
\hline
\end{tabular}


Table 1 Brief summary of included studies (Continued)

\begin{tabular}{|c|c|c|c|c|c|c|}
\hline \multirow[t]{2}{*}{ Article } & \multicolumn{2}{|l|}{ Study } & \multirow{2}{*}{$\begin{array}{l}\text { Participants } \\
\text { Number/age/sex }\end{array}$} & \multirow{2}{*}{$\begin{array}{l}\text { Reasons for using } \\
\text { cannabis medically }\end{array}$} & \multirow{2}{*}{$\begin{array}{l}\text { Reported effects and } \\
\text { perceptions of } \\
\text { medical cannabis }\end{array}$} & \multirow[t]{2}{*}{ Funding } \\
\hline & $\begin{array}{l}\text { Objectives/design: } \\
\text { data source; } \\
\text { recruitment }\end{array}$ & $\begin{array}{l}\text { Location } \\
\text { /period, legality }{ }^{1}\end{array}$ & & & & \\
\hline & $\begin{array}{l}\text { patients enrolled in the } \\
\text { Canadian Marihuana for } \\
\text { Medical Purposes } \\
\text { regulations. } \\
\text { Quantitative: Online } \\
\text { cross-sectional survey; } \\
\text { recruitment through a } \\
\text { licensed producer of } \\
\text { cannabis. }\end{array}$ & $\begin{array}{l}\text { use (Marihuana } \\
\text { for Medical } \\
\text { Purposes } \\
\text { Regulations *). }\end{array}$ & $\begin{array}{l}\text { Purposes Regulations). } \\
40 \text { (20-77) years. } \\
73 \% \text { men. }\end{array}$ & $\begin{array}{l}\text { arthritis, 5\% headache. } \\
\text { Most highly endorsed } \\
\text { symptoms: } \\
\text { 73\% chronic pain, 60\%, } \\
\text { stress, 57\% insomnia, } \\
\text { 46\% depression, 32\% } \\
\text { headache. }\end{array}$ & their symptoms. & Chronic Disease \\
\hline $\begin{array}{l}\text { Lynch et al. } \\
2006\end{array}$ & $\begin{array}{l}\text { To describe medical } \\
\text { cannabis users. } \\
\text { Quantitative: Structured } \\
\text { follow-up questionnaire; } \\
\text { recruitment of patients } \\
\text { followed at a tertiary } \\
\text { care pain management } \\
\text { center. }\end{array}$ & $\begin{array}{l}\text { Nova Scotia, } \\
\text { Canada. } \\
\text { 2001-2005, legal } \\
\text { medical cannabis } \\
\text { use (Marihuana } \\
\text { Medical Access } \\
\text { Regulations } \\
\text { Marihuana } \\
\text { Medical Access } \\
\text { Regulations *). }\end{array}$ & $\begin{array}{l}30 \text { qualified medical } \\
\text { cannabis users } \\
\text { (Marihuana Medical } \\
\text { Access Regulations). } \\
45 \text { ( } 31-61 \text { ) years. } \\
60 \% \text { men. }\end{array}$ & $\begin{array}{l}\text { Chronic severe pain } \\
\text { that had not responded } \\
\text { to traditional } \\
\text { approaches: } \\
\text { 47\% neuropathic pain } \\
13 \% \text { low back pain } \\
\text { 10\% arthritis. }\end{array}$ & $\begin{array}{l}\text { 93\% reported moderate } \\
\text { or greater pain relief. } \\
95 \% \text { reported subjective } \\
\text { improvement in } \\
\text { function. } \\
\text { No serious adverse } \\
\text { events reported. }\end{array}$ & $\mathrm{N} / \mathrm{A}$ \\
\hline $\begin{array}{l}\text { Nunberg et al. } \\
2011 \text { and } \\
\text { Reinarman } \\
\text { et al. } 2011\end{array}$ & $\begin{array}{l}\text { To describe medical } \\
\text { cannabis users: } \\
\text { demographics; } \\
\text { symptoms; physician } \\
\text { evaluations; } \\
\text { conventional } \\
\text { treatments tried; use } \\
\text { practices. } \\
\text { Quantitative: Physician } \\
\text { records and patients' } \\
\text { questionnaire; } \\
\text { recruitment through } \\
\text { nine medical cannabis } \\
\text { clinics. }\end{array}$ & $\begin{array}{l}\text { California, USA. } \\
\text { June-August } \\
\text { 2006, legal } \\
\text { medical cannabis } \\
\text { use. }\end{array}$ & $\begin{array}{l}1746 \text { medical cannabis } \\
\text { applicants. } \\
33 \% \geq 45 \text { years. } \\
75 \% \text { men. }\end{array}$ & $\begin{array}{l}82.6 \% \text { report using } \\
\text { medical cannabis to } \\
\text { relieve pain. } \\
58.2 \% \text { diagnosed with } \\
\text { chronic pain disorders, } \\
\text { including: } \\
26 \% \text { low back pain } \\
18 \% \text { arthritis } \\
2 \% \text { fibromyalgia. }\end{array}$ & $\begin{array}{l}\text { Patients typically report } \\
\text { at least one therapeutic } \\
\text { benefit: } \\
83 \% \text { relief of pain } \\
41 \% \text { muscle spasms } \\
41 \% \text { headache } \\
38 \% \text { anxiety } \\
28 \% \text { nausea and } \\
\text { vomiting } \\
26 \% \text { depression. }\end{array}$ & $\begin{array}{l}\text { (Mixed funding) } \\
\text { Research grant } \\
\text { RAND Corporation; } \\
\text { Non- } \\
\text { governmental } \\
\text { organization } \\
\text { funding } \\
\text { Cannabis } \\
\text { "industry" } \\
\text { MediCann; Private } \\
\text { Foundation } \\
\text { Rosenbaum } \\
\text { Foundation }\end{array}$ \\
\hline $\begin{array}{l}\text { Ogborne et al. } \\
2000\end{array}$ & $\begin{array}{l}\text { To explore reasons for } \\
\text { medical cannabis use; } \\
\text { medical cannabis effects; } \\
\text { methods and patterns of } \\
\text { use; experiences with } \\
\text { physicians; encounters } \\
\text { with the law. } \\
\text { Qualitative: Interview; } \\
\text { recruitment through } \\
\text { advertisements in } \\
\text { newspapers and on } \\
\text { bulletin boards at an } \\
\text { Addiction Research } \\
\text { Foundation and at } \\
\text { different town locations } \\
\text { (bookstores, grocery } \\
\text { stores, restaurants, } \\
\text { laundromats, etc). }\end{array}$ & $\begin{array}{l}\text { Toronto, Canada. } \\
\text { Before the } 2001 \\
\text { Marihuana } \\
\text { Medical Access } \\
\text { Program. }\end{array}$ & $\begin{array}{l}50 \text { self-identified med- } \\
\text { ical cannabis users. } \\
38(26-57) \text { years. } \\
66 \% \text { men. }\end{array}$ & $\begin{array}{l}22 \% \text { HIV/AIDS-related } \\
\text { symptoms } \\
14 \% \text { chronic/recurrent } \\
\text { pain due to injury of } \\
\text { unknown origin } \\
12 \% \text { depression } \\
2 \% \text { arthritis. }\end{array}$ & $\begin{array}{l}\text { medical cannabis } \\
\text { described as superior to } \\
\text { other treatments. } \\
\text { Reported lethargy, } \\
\text { apathy, cough or throat } \\
\text { irritation from smoking, } \\
\text { thirst, loss of } \\
\text { concentration, short- } \\
\text { term memory loss, para- } \\
\text { noia, and depression. }\end{array}$ & N/A \\
\hline $\begin{array}{l}\text { Pedersen \& } \\
\text { Sandberg } \\
2013\end{array}$ & $\begin{array}{l}\text { To investigate the } \\
\text { medical motives of } \\
\text { Norwegian cannabis } \\
\text { users. } \\
\text { Qualitative: Semi- } \\
\text { structured interviews; } \\
\text { recruitment through } \\
\text { internet advertisements, } \\
\text { authors' own social net- } \\
\text { works, among students } \\
\text { at the University of } \\
\text { Oslo, and from organi- }\end{array}$ & $\begin{array}{l}\text { Norway. } \\
\text { 2006-2010, illegal. }\end{array}$ & $\begin{array}{l}100 \text { long-term canna- } \\
\text { bis users ( } 25 \text { stated ex- } \\
\text { plicitly they used } \\
\text { cannabis medically). } \\
20-62 \text { years. } \\
88 \% \text { men. }\end{array}$ & $\begin{array}{l}\text { Cannabis was used } \\
\text { therapeutically for } \\
\text { conditions such as } \\
\text { multiple sclerosis, } \\
\text { attention deficit } \\
\text { hyperactivity disorder } \\
\text { and rheumatism, as } \\
\text { well as for quality of life } \\
\text { conditions such as } \\
\text { quality of sleep, } \\
\text { relaxation and } \\
\text { wellbeing. }\end{array}$ & $\begin{array}{l}\text { Cannabis typically } \\
\text { described as useful for } \\
\text { treating stress, insomnia } \\
\text { and pain, as well as for } \\
\text { relaxation. }\end{array}$ & $\begin{array}{l}\text { Research grant } \\
\text { Research Council } \\
\text { of Norway }\end{array}$ \\
\hline
\end{tabular}


Table 1 Brief summary of included studies (Continued)

\begin{tabular}{|c|c|c|c|c|c|c|}
\hline \multirow[t]{2}{*}{ Article } & \multicolumn{2}{|l|}{ Study } & \multirow{2}{*}{$\begin{array}{l}\text { Participants } \\
\text { Number/age/sex }\end{array}$} & \multirow{2}{*}{$\begin{array}{l}\text { Reasons for using } \\
\text { cannabis medically }\end{array}$} & \multirow{2}{*}{$\begin{array}{l}\text { Reported effects and } \\
\text { perceptions of } \\
\text { medical cannabis }\end{array}$} & \multirow[t]{2}{*}{ Funding } \\
\hline & $\begin{array}{l}\text { Objectives/design: } \\
\text { data source; } \\
\text { recruitment }\end{array}$ & $\begin{array}{l}\text { Location } \\
\text { /period, legality }^{1}\end{array}$ & & & & \\
\hline $\begin{array}{l}\text { Perron et al. } \\
2015\end{array}$ & $\begin{array}{l}\text { To better elucidate, } \\
\text { among MC users with } \\
\text { and without concurrent } \\
\text { use of prescription pain } \\
\text { medication (PPM): } \\
\text { patterns of alcohol and } \\
\text { other drug use; } \\
\text { functioning; perceived } \\
\text { efficacy of pain } \\
\text { treatments. } \\
\text { Quantitative: } \\
\text { Questionnaires; } \\
\text { recruitment via a survey } \\
\text { conducted among } \\
\text { persons seeking } \\
\text { medical cannabis } \\
\text { certification or } \\
\text { recertification at an } \\
\text { medical cannabis } \\
\text { certification clinic. }\end{array}$ & $\begin{array}{l}\text { Michigan, USA. } \\
\text { Legal medical } \\
\text { cannabis use. }\end{array}$ & $\begin{array}{l}273 \text { adults reporting } \\
\text { past-month cannabis } \\
\text { use for pain-related } \\
\text { purposes (subsample } \\
\text { of Ilgen et al.'s } 2013 \\
\text { study). } \\
40.3 \pm 12.5 \text { years. } \\
69 \% \text { men. }\end{array}$ & $\begin{array}{l}\text { Subset of subjects who } \\
\text { endorsed using } \\
\text { cannabis in the past } \\
\text { month specifically for } \\
\text { pain reduction. }\end{array}$ & $\begin{array}{l}\text { Prescription pain } \\
\text { medication (PPM) users } \\
\text { perceived cannabis as } \\
\text { more efficacious than } \\
\text { PPMs. }\end{array}$ & $\begin{array}{l}\text { Research grant } \\
\text { National Institute } \\
\text { on Drug Abuse } \\
\text { grant }\end{array}$ \\
\hline $\begin{array}{l}\text { Piper et al. } \\
2017\end{array}$ & $\begin{array}{l}\text { To provide an in-depth } \\
\text { qualitative exploration } \\
\text { of patient perspectives } \\
\text { on the strengths and } \\
\text { limitations of medical } \\
\text { cannabis. } \\
\text { Online survey with } \\
\text { open-ended questions; } \\
\text { recruitment via medical } \\
\text { cannabis dispensaries. }\end{array}$ & $\begin{array}{l}\text { Maine, Vermont, } \\
\text { and Rhode Island, } \\
\text { USA. } \\
\text { 2015-2016 } \\
\text { (chronic pain was } \\
\text { not a condition to } \\
\text { become part of } \\
\text { the Vermont } \\
\text { registry). }\end{array}$ & $\begin{array}{l}984 \text { members of } \\
\text { medical cannabis } \\
\text { dispensaries. } \\
49.1 \pm 0.5 \text { years. } \\
47 \% \text { men. }\end{array}$ & $\begin{array}{l}64 \% \text { reported a } \\
\text { diagnosis of chronic } \\
\text { pain:91\% back/neck } \\
\text { pain30\% neuropathic } \\
\text { pain } 23 \% \text { postsurgical } \\
\text { pain } 22 \% \text { abdominal } \\
\text { pain } 20 \% \text { chronic pain } \\
\text { after trauma/injury. }\end{array}$ & $\begin{array}{l}75 \% \text { relief of symptoms. } \\
\text { Reported benefits: pain } \\
\text { relief, better sleep, safe/ } \\
\text { natural (limited } \\
\text { addictive potential), } \\
\text { quality of life, } \\
\text { functionality. } \\
\text { Negative themes: } \\
\text { respiratory effects, } \\
\text { increased appetite, } \\
\text { cognitive (decrease } \\
\text { ability to concentrate, } \\
\text { non-alert feeling...). }\end{array}$ & $\begin{array}{l}\text { (Mixed) } \\
\text { Nonprofit } \\
\text { organization } \\
\text { funding } \\
\text { Center for Wellness } \\
\text { Leadership } \\
\text { Local resource } \\
\text { funding } \\
\text { Wellness } \\
\text { Connection of } \\
\text { Maine (Burstein, } \\
\text { 2015); Research } \\
\text { grant } \\
\text { National Institute } \\
\text { of Drug Abuse }\end{array}$ \\
\hline Reiman 2009 & $\begin{array}{l}\text { To examine drug and } \\
\text { alcohol use, and the } \\
\text { occurrence of } \\
\text { substitution among } \\
\text { medical cannabis users. } \\
\text { Quantitative: Survey } \\
\text { data collected at a } \\
\text { medical cannabis } \\
\text { dispensing collective; } \\
\text { recruitment through an } \\
\text { medical cannabis } \\
\text { dispensing collective. }\end{array}$ & $\begin{array}{l}\text { California, USA. } \\
\text { Legal medical } \\
\text { cannabis use. }\end{array}$ & $\begin{array}{l}350 \text { medical cannabis } \\
\text { users } \\
39(18-81) \text { years. } \\
68 \% \text { men. }\end{array}$ & $\begin{array}{l}52 \% \text { use cannabis for a } \\
\text { pain related condition, } \\
\text { including } 45 \% \text { who } \\
\text { used it against pain } \\
\text { resulting from an } \\
\text { alcohol related } \\
\text { accident. } \\
75 \% \text { use cannabis for a } \\
\text { mental health issue. }\end{array}$ & $\begin{array}{l}65 \% \text { use medical } \\
\text { cannabis as a substitute } \\
\text { for alcohol, illicit or licit } \\
\text { drugs with less adverse } \\
\text { side effects. }\end{array}$ & $\mathrm{N} / \mathrm{A}$ \\
\hline $\begin{array}{l}\text { Reiman et al. } \\
2017\end{array}$ & $\begin{array}{l}\text { To gather the } \\
\text { impressions of patients } \\
\text { who have used } \\
\text { cannabis on how it } \\
\text { compares with pain } \\
\text { medications. } \\
\text { Quantitative: Cross- } \\
\text { sectional survey; recruit- } \\
\text { ment through e-mails } \\
\text { addressed to medical } \\
\text { cannabis patients of an } \\
\text { medical cannabis pa- } \\
\text { tient database (67,422 } \\
\text { patients). }\end{array}$ & California, USA. & $\begin{array}{l}2897 \text { medical cannabis } \\
\text { respondents seeking } \\
\text { medical cannabis } \\
\text { certification. } \\
\geq 20 \text { years. } \\
55 \% \text { men. }\end{array}$ & $\begin{array}{l}63 \% \text { pain-related condi- } \\
\text { tions including back } \\
\text { pain and arthritis. }\end{array}$ & $\begin{array}{l}\text { Respondents } \\
\text { overwhelmingly } \\
\text { reported that cannabis } \\
\text { provided relief on par } \\
\text { with their other } \\
\text { medications, but } \\
\text { without the unwanted } \\
\text { side effects. }\end{array}$ & N/A \\
\hline $\begin{array}{l}\text { Sagy et al. } \\
2019\end{array}$ & $\begin{array}{l}\text { To investigate the } \\
\text { characteristics, safety } \\
\text { and effectiveness of } \\
\text { medical cannabis in } \\
\text { fibromyalgia over a } \\
\text { period of } 6 \text { months. }\end{array}$ & $\begin{array}{l}\text { Israel. } \\
\text { 2015-2017, legal } \\
\text { medical cannabis } \\
\text { use. }\end{array}$ & $\begin{array}{l}367 \text { fibromyalgia } \\
\text { patients, qualified } \\
\text { medical cannabis } \\
\text { users. } \\
52.9( \pm 15.1) \text { years. } \\
18 \% \text { men. }\end{array}$ & 100\% fibromyalgia. & $\begin{array}{l}\text { Overall pain intensity } \\
\text { assessed by NRS } \\
\text { reduced from a median } \\
\text { of } 9.0 \text { at baseline to } 5.0 \\
\text { after } 6 \text { months of } \\
\text { medical cannabis }\end{array}$ & N/A \\
\hline
\end{tabular}


Table 1 Brief summary of included studies (Continued)

\begin{tabular}{|c|c|c|c|c|c|c|}
\hline \multirow[t]{2}{*}{ Article } & \multicolumn{2}{|l|}{ Study } & \multirow{2}{*}{$\begin{array}{l}\text { Participants } \\
\text { Number/age/sex }\end{array}$} & \multirow{2}{*}{$\begin{array}{l}\text { Reasons for using } \\
\text { cannabis medically }\end{array}$} & \multirow{2}{*}{$\begin{array}{l}\text { Reported effects and } \\
\text { perceptions of } \\
\text { medical cannabis }\end{array}$} & \multirow[t]{2}{*}{ Funding } \\
\hline & $\begin{array}{l}\text { Objectives/design: } \\
\text { data source; } \\
\text { recruitment }\end{array}$ & $\begin{array}{l}\text { Location } \\
\text { /period, legality }{ }^{1}\end{array}$ & & & & \\
\hline & $\begin{array}{l}\text { Quantitative: } \\
\text { Questionnaire; } \\
\text { recruitment via medical } \\
\text { cannabis provider. }\end{array}$ & & & & $\begin{array}{l}\text { treatment }(P<0.001) \text {. } \\
\text { Side effects: dizziness } \\
(7.9 \%) \text {, dry mouth } \\
(6.7 \%), \text { nausea/vomiting } \\
(5.4 \%) \text {, hyperactivity } \\
(5.5 \%) \text {, increased } \\
\text { appetite }(3.8 \%) \text {. }\end{array}$ & \\
\hline $\begin{array}{l}\text { Schnelle et al. } \\
1999\end{array}$ & $\begin{array}{l}\text { Quantitative: } \\
\text { questionnaire; } \\
\text { recruitment via an } \\
\text { medical cannabis } \\
\text { association. }\end{array}$ & $\begin{array}{l}\text { Germany, Austria } \\
\text { and Switzerland. } \\
\text { 1998-1999. }\end{array}$ & $\begin{array}{l}128 \text { qualified medical } \\
\text { cannabis users. } \\
37.5 \pm 9.6 \text { y } \\
68 \% \text { men }\end{array}$ & $\begin{array}{l}\text { 12\% depression } \\
11 \% \text { multiple sclerosis } \\
9 \% \text { HIV infection } \\
5 \% \text { back pain. }\end{array}$ & $\begin{array}{l}\text { Symptoms } \\
\text { improvement from } \\
\text { much }(72.2 \%) \text {, to none } \\
(4.8 \%) \text {. } \\
1.6 \% \text { experienced } \\
\text { worsening of } \\
\text { symptoms. } \\
70.8 \% \text { experiences no } \\
\text { adverse effects. }\end{array}$ & N/A \\
\hline $\begin{array}{l}\text { Sexton et al. } \\
2016\end{array}$ & $\begin{array}{l}\text { To collect } \\
\text { epidemiologic data to } \\
\text { inform medical practice, } \\
\text { research, and policy to } \\
\text { provoke discussion } \\
\text { about the discrepancies } \\
\text { between medico-legal } \\
\text { recommendations and } \\
\text { patient-reported } \\
\text { outcomes. } \\
\text { Quantitative: Cross- } \\
\text { sectional online survey); } \\
\text { recruitment through } \\
\text { links posted on Univer- } \\
\text { sity (Bastyr University } \\
\text { California (US)) websites, } \\
\text { social media and canna- } \\
\text { bis dispensaries. }\end{array}$ & $\begin{array}{l}\text { Respondents } \\
\text { came from } 18 \\
\text { countries, with } \\
\text { the USA (78\%), } \\
\text { the UK (6\%), and } \\
\text { Canada (3\%) } \\
\text { being the most } \\
\text { represented. } \\
2013-2016 . \\
\text { Legality varies } \\
\text { across countries. }\end{array}$ & $\begin{array}{l}\text { Convenience sample } \\
\text { of } 1429 \text { self-identified } \\
\text { medical cannabis } \\
\text { users. } \\
36.3 \pm 14 \text { (15-80) years. } \\
55 \% \text { men. }\end{array}$ & $\begin{array}{l}\text { 61\% pain } \\
58 \% \text { anxiety } \\
50 \% \text { depression } \\
35.5 \% \text { headache/ } \\
\text { migraine } \\
27 \% \text { nausea } \\
18 \% \text { muscle spasticity } \\
17 \% \text { arthritis } \\
15 \% \text { irritable bowel } \\
11.5 \% \text { intractable pain. }\end{array}$ & $\begin{array}{l}\text { On average, participants } \\
\text { reported an } 86 \% \\
\text { reduction in symptoms. }\end{array}$ & $\begin{array}{l}\text { Research grant } \\
\text { NIH NCCAM } \\
\text { K01ATTA (Ste-Marie } \\
\text { et al., 2016) }\end{array}$ \\
\hline $\begin{array}{l}\text { Shah et al. } \\
2017\end{array}$ & $\begin{array}{l}\text { To examine clinical and } \\
\text { treatment } \\
\text { characteristics for } \\
\text { patients who are } \\
\text { admitted to a 3-week } \\
\text { outpatient inter- } \\
\text { disciplinary chronic pain } \\
\text { rehabilitation program. } \\
\text { Quantitative: Self-report } \\
\text { questionnaire and chart } \\
\text { review; recruitment of } \\
\text { patients admitted to a } \\
\text { 3-week outpatient inter- } \\
\text { disciplinary chronic pain } \\
\text { rehabilitation program. }\end{array}$ & $\begin{array}{l}\text { The USA. } \\
\text { March-December } \\
2015 . \\
\text { Not reported }\end{array}$ & $\begin{array}{l}24 \text { patients with THC } \\
\text { positive urine test } \\
\text { participating to a pain } \\
\text { rehabilitation program. } \\
45.4 \pm 15.3 \text { years. } \\
42 \% \text { men. }\end{array}$ & Chronic pain. & Not reported & $\mathrm{N} / \mathrm{A}$ \\
\hline $\begin{array}{l}\text { Shiplo et al. } \\
2016\end{array}$ & $\begin{array}{l}\text { To examine modes of } \\
\text { medical cannabis } \\
\text { delivery following } \\
\text { regulatory changes in } \\
2014 . \\
\text { Quantitative: Online } \\
\text { cross-sectional survey; } \\
\text { recruitment via nine } \\
\text { Health Canada licenced } \\
\text { medical cannabis } \\
\text { producers. }\end{array}$ & $\begin{array}{l}\text { Canada. } \\
\text { April-June } 2015 . \\
\text { Legal medical } \\
\text { cannabis use. }\end{array}$ & $\begin{array}{l}\text { Convenience sample } \\
\text { of } 364 \text { qualified } \\
\text { medical cannabis } \\
\text { users. } \\
40.8 \pm 12.6 \text { years. } \\
58 \% \text { men. }\end{array}$ & $\begin{array}{l}45 \% \text { for pain relief } \\
\text { (chronic pain and } \\
\text { fibromyalgia) } \\
15 \% \text { mental health } \\
10 \% \text { central nervous } \\
\text { system. }\end{array}$ & not reported & $\begin{array}{l}\text { Research grant } \\
\text { Canadian Institute } \\
\text { of Health Research } \\
\text { (CIHR) Training } \\
\text { Grant Program in } \\
\text { Population } \\
\text { Intervention for } \\
\text { Chronic Disease } \\
\text { Prevention }\end{array}$ \\
\hline $\begin{array}{l}\text { Ste-Marie } \\
\text { et al. } 2012\end{array}$ & $\begin{array}{l}\text { To document the self- } \\
\text { identified prevalence of } \\
\text { cannabinoid use in } \\
\text { fibromyalgia patients } \\
\text { seen in a fibromyalgia } \\
\text { clinic. }\end{array}$ & $\begin{array}{l}\text { Montreal, Canada. } \\
\text { 2005-2010. } \\
\text { Legal medical } \\
\text { cannabis use. }\end{array}$ & $\begin{array}{l}59 \text { medical cannabis } \\
\text { users with a diagnosis } \\
\text { of fibromyalgia. } 24 \% \\
\text { used prescription } \\
\text { cannabinoids. } \\
45 \pm 10 \text { y }\end{array}$ & $\begin{array}{l}\text { Fibromyalgia (61\%) or } \\
\text { regional pain syndrome } \\
\text { and spinal pain, } \\
\text { rheumatic disease, } \\
\text { neurologic condition. }\end{array}$ & Not reported & $\begin{array}{l}\text { Research grant } \\
\text { Louise and Alan } \\
\text { Edwards } \\
\text { Foundation }\end{array}$ \\
\hline
\end{tabular}


Table 1 Brief summary of included studies (Continued)

\begin{tabular}{|c|c|c|c|c|c|c|}
\hline \multirow[t]{2}{*}{ Article } & \multicolumn{2}{|l|}{ Study } & \multirow{2}{*}{$\begin{array}{l}\text { Participants } \\
\text { Number/age/sex }\end{array}$} & \multirow{2}{*}{$\begin{array}{l}\text { Reasons for using } \\
\text { cannabis medically }\end{array}$} & \multirow{2}{*}{$\begin{array}{l}\text { Reported effects and } \\
\text { perceptions of } \\
\text { medical cannabis }\end{array}$} & \multirow[t]{2}{*}{ Funding } \\
\hline & $\begin{array}{l}\text { Objectives/design: } \\
\text { data source; } \\
\text { recruitment }\end{array}$ & $\begin{array}{l}\text { Location } \\
\text { /period, legality }\end{array}$ & & & & \\
\hline & $\begin{array}{l}\text { Qualitative: } \\
\text { Retrospective chart } \\
\text { review; recruitment via } \\
\text { a tertiary care pain } \\
\text { center. }\end{array}$ & & $33 \%$ men. & & & \\
\hline $\begin{array}{l}\text { Ste-Marie } \\
\text { et al. } 2016\end{array}$ & $\begin{array}{l}\text { To examine the } \\
\text { prevalence of cannabis } \\
\text { use among } \\
\text { rheumatology patients. } \\
\text { To compare the clinical } \\
\text { characteristics of } \\
\text { medical cannabis users } \\
\text { and nonusers. } \\
\text { Quantitative: Cross- } \\
\text { sectional survey (ques- } \\
\text { tionnaires); recruitment } \\
\text { via an university- } \\
\text { affiliated community } \\
\text { rheumatology clinic. }\end{array}$ & $\begin{array}{l}\text { Ontario, Canada. } \\
\text { April-May } 2014 . \\
\text { Legal medical } \\
\text { cannabis use. }\end{array}$ & $\begin{array}{l}28 \text { current medical } \\
\text { cannabis users. } \\
52.7 \pm 13.6 \text { years. } \\
43 \% \text { men. } \\
15 \text { previous medical } \\
\text { cannabis users, } 62.8 \pm \\
14.4 \text { y, } 26 \% \text { men. }\end{array}$ & $\begin{array}{l}\text { Specific rheumatic } \\
\text { disease : } \\
54 \% \text { osteoarthritis or } \\
\text { spinal pain } \\
32 \% \text { inflammatory } \\
\text { arthritis } \\
\text { 18\% fibromyalgia. }\end{array}$ & $\begin{array}{l}\text { Medical cannabis } \\
\text { reported to relieve pain, } \\
\text { anxiety, nausea, } \\
\text { improve sleep and } \\
\text { appetite. }\end{array}$ & $\begin{array}{l}\text { Research grant } \\
\text { Louise and Alan } \\
\text { Edwards } \\
\text { Foundation }\end{array}$ \\
\hline $\begin{array}{l}\text { Swift et al. } \\
2005\end{array}$ & $\begin{array}{l}\text { To learn more about: } \\
\text { patterns of use; } \\
\text { experiences and } \\
\text { concerns; interest in } \\
\text { participating in a } \\
\text { medical cannabis trial. } \\
\text { Quantitative: Mailed } \\
\text { questionnaires; } \\
\text { recruitment through } \\
\text { opportunistic media } \\
\text { stories in newspapers, } \\
\text { on radio and television. }\end{array}$ & $\begin{array}{l}\text { Australia. } \\
\text { 2003-2004. } \\
\text { Illegal. }\end{array}$ & $\begin{array}{l}128 \text { medical cannabis } \\
\text { users } \\
\text { Median } 45 \text { (24-88) } \\
\text { years. } \\
63 \% \text { men. }\end{array}$ & $\begin{array}{l}\text { Condition: } \\
\text { 60\% depression } \\
\text { 53\% chronic pain } \\
\text { 38\% arthritis. }\end{array}$ & $\begin{array}{l}86 \% \text { reported great } \\
\text { relief from cannabis. } \\
\text { Typically perceived as } \\
\text { superior to other } \\
\text { medications in terms of } \\
\text { undesirable effects, and } \\
\text { the extent of relief } \\
\text { provided. } \\
15 \% \text { had stopped, } 16 \% \\
\text { disliked the side effects } \\
\text { or route of use (each 3/ } \\
\text { 19). }\end{array}$ & $\mathrm{N} / \mathrm{A}$ \\
\hline $\begin{array}{l}\text { Troutt \& } \\
\text { DiDonato, } \\
2015\end{array}$ & $\begin{array}{l}\text { To examine medical } \\
\text { cannabis users: } \\
\text { characteristics; } \\
\text { perceptions; behaviors. } \\
\text { To learn about } \\
\text { experiences with } \\
\text { cannabis before } \\
\text { legalization. } \\
\text { Quantitative: } \\
\text { Anonymous online } \\
\text { survey; recruitment: via } \\
\text { four medical cannabis } \\
\text { dispensaries. }\end{array}$ & $\begin{array}{l}\text { Arizona, USA. } \\
\text { After the } 2012 \\
\text { Arizona } \\
\text { Department of } \\
\text { Health Services } \\
\text { Medical Marijuana } \\
\text { Rules. }\end{array}$ & $\begin{array}{l}367 \text { patients recruited } \\
\text { from medical cannabis } \\
\text { dispensaries. } \\
45.78 \pm 13.76(18-83) \\
\text { years. } \\
64 \% \text { men. }\end{array}$ & $\begin{array}{l}87 \% \text { chronic pain } \\
24.5 \% \text { arthritis } \\
11 \% \text { osteoarthritis } \\
7 \% \text { fibromyalgia. }\end{array}$ & $\begin{array}{l}70 \% \text { experienced a lot } \\
\text { of or almost complete } \\
\text { relief. }\end{array}$ & N/A \\
\hline $\begin{array}{l}\text { Walsh et al. } \\
2013 \text { and } \\
\text { Belle-Isle et al. } \\
2014\end{array}$ & $\begin{array}{l}\text { To examine: cannabis } \\
\text { use history; medical } \\
\text { conditions and } \\
\text { symptoms; patterns of } \\
\text { use; modes of access; } \\
\text { perceived effectiveness. } \\
\text { Quantitative: Survey } \\
\text { (online or at a cannabis } \\
\text { dispensary); recruitment } \\
\text { through local medical } \\
\text { cannabis dispensaries } \\
\text { and national } \\
\text { organizations that assist } \\
\text { medical cannabis users. }\end{array}$ & $\begin{array}{l}\text { British Columbia, } \\
\text { Canada. } \\
\text { 2011-2012. } \\
\text { Legal medical } \\
\text { cannabis use. }\end{array}$ & $\begin{array}{l}628 \text { self-identified } \\
\text { current medical canna- } \\
\text { bis users. } \\
39.1 \pm 13.1 \text { years. } \\
71 \% \text { men. }\end{array}$ & $\begin{array}{l}\text { Pain, including chronic, } \\
\text { spinal and non-spinal } \\
\text { pain, arthritis (82\%), } \\
\text { anxiety, and sleep } \\
\text { problems. }\end{array}$ & $\begin{array}{l}\text { Cannabis perceived to } \\
\text { provide effective } \\
\text { symptoms relief: } \\
72 \% \text { reported medical } \\
\text { cannabis always helpful, } \\
24 \% \text { often helpful. }\end{array}$ & $\begin{array}{l}\text { Research grant } \\
\text { UBC Institute for } \\
\text { Healthy Living and } \\
\text { Chronic Disease } \\
\text { Prevention }\end{array}$ \\
\hline $\begin{array}{l}\text { Ware et al. } \\
2003\end{array}$ & $\begin{array}{l}\text { To determine current } \\
\text { prevalence of medical } \\
\text { cannabis in chronic } \\
\text { non-cancer pain; esti- } \\
\text { mate the dose size and } \\
\text { frequency of cannabis } \\
\text { use; describe main } \\
\text { symptoms for which }\end{array}$ & $\begin{array}{l}\text { Nova Scotia, } \\
\text { Canada. } \\
\text { June to July } 2001 . \\
\text { Legal medical } \\
\text { cannabis use. }\end{array}$ & $\begin{array}{l}09 \text { chronic non-cancer } \\
\text { pain patients.35\% had } \\
\text { ever used cannabis, } \\
15 \% \text { have used canna- } \\
\text { bis for pain relief, and } \\
10 \% \text { were current MC } \\
\text { users for pain relief. }\end{array}$ & $\begin{array}{l}\text { Of MC users: } \\
50 \% \text { trauma/surgery } \\
6 \% \text { arthritis } \\
6 \% \text { multiple sclerosis. }\end{array}$ & $\begin{array}{l}\text { Improved pain, sleep } \\
\text { and mood. } \\
78 \% \text { of medical } \\
\text { cannabis users reported } \\
\text { at least moderate relief } \\
\text { of pain. } \\
25 \% \text { reported no side } \\
\text { effects, } 37 \% \text { very mild, }\end{array}$ & $\begin{array}{l}\text { (Mixed) } \\
\text { University funding } \\
\text { *Faculty of } \\
\text { Medicine } \\
\text { *Department of } \\
\text { Anesthesia; Non- } \\
\text { governmental } \\
\text { organization }\end{array}$ \\
\hline
\end{tabular}


Table 1 Brief summary of included studies (Continued)

\begin{tabular}{|c|c|c|c|c|c|c|}
\hline \multirow[t]{2}{*}{ Article } & \multicolumn{2}{|l|}{ Study } & \multirow{2}{*}{$\begin{array}{l}\text { Participants } \\
\text { Number/age/sex }\end{array}$} & \multirow{2}{*}{$\begin{array}{l}\text { Reasons for using } \\
\text { cannabis medically }\end{array}$} & \multirow{2}{*}{$\begin{array}{l}\text { Reported effects and } \\
\text { perceptions of } \\
\text { medical cannabis }\end{array}$} & \multirow[t]{2}{*}{ Funding } \\
\hline & $\begin{array}{l}\text { Objectives/design: } \\
\text { data source; } \\
\text { recruitment }\end{array}$ & $\begin{array}{l}\text { Location } \\
\text { /period, legality }^{1}\end{array}$ & & & & \\
\hline & $\begin{array}{l}\text { relief was sought. } \\
\text { Quantitative: Cross- } \\
\text { sectional survey; recruit- } \\
\text { ment of all patients en- } \\
\text { tering the ambulatory } \\
\text { pain management unit } \\
\text { of the Queen Elizabeth } \\
\text { II Health Sciences } \\
\text { Center. }\end{array}$ & & & & $\begin{array}{l}\text { 28\% moderate, } 9 \% \\
\text { strong side effects, no } \\
\text { severe side effects. }\end{array}$ & $\begin{array}{l}\text { funding } \\
\text { Research-based } \\
\text { pharmaceutical } \\
\text { companies }\end{array}$ \\
\hline $\begin{array}{l}\text { Webb \& } \\
\text { Webb } 2014\end{array}$ & $\begin{array}{l}\text { To discover the benefits } \\
\text { and adverse effects } \\
\text { perceived by medical } \\
\text { cannabis users, } \\
\text { especially with regards } \\
\text { to chronic pain. } \\
\text { Quantitative: Survey } \\
\text { (questionnaires); } \\
\text { recruitment via } \\
\text { questionnaires hand- } \\
\text { delivered to medical } \\
\text { cannabis certified pa- } \\
\text { tients re-applying for } \\
\text { certification. }\end{array}$ & $\begin{array}{l}\text { Hawaii, USA. } \\
\text { 2010-2011. } \\
\text { Legal MC use. }\end{array}$ & $\begin{array}{l}94 \text { patients re-applying } \\
\text { for medical cannabis } \\
\text { certification. } \\
49.3 \text { years. }\end{array}$ & $\begin{array}{l}97 \% \text { used cannabis } \\
\text { primarily for chronic } \\
\text { pain. }\end{array}$ & $\begin{array}{l}64 \% \text { relative decrease in } \\
\text { average pain. } \\
71 \% \text { reported no } \\
\text { adverse effects, } 6 \% \\
\text { reported a cough or } \\
\text { throat irritation. }\end{array}$ & $\mathrm{N} / \mathrm{A}$ \\
\hline $\begin{array}{l}\text { Zaller et al. } \\
2015\end{array}$ & $\begin{array}{l}\text { To characterize socio- } \\
\text { demographics and rea- } \\
\text { sons for medical canna- } \\
\text { bis use among } \\
\text { dispensary patients. } \\
\text { Quantitative: Cross- } \\
\text { sectional survey (ques- } \\
\text { tionnaires); recruitment } \\
\text { through Compassion } \\
\text { Centers of the Depart- } \\
\text { ment of Health. }\end{array}$ & $\begin{array}{l}\text { Rhode Island, } \\
\text { USA. } \\
\text { After the } 2013 \\
\text { authorization for } \\
\text { medical cannabis } \\
\text { dispensaries. }\end{array}$ & $\begin{array}{l}200 \text { qualified medical } \\
\text { cannabis users. } \\
\text { Median } 41 \text { (18-76) } \\
\text { years. } \\
73 \% \text { men. }\end{array}$ & $\begin{array}{l}\text { The most common } \\
\text { reason for medical } \\
\text { cannabis use was } \\
\text { chronic pain } \\
\text { management. }\end{array}$ & $\begin{array}{l}\text { Most participants report } \\
\text { that medical cannabis } \\
\text { improves their pain } \\
\text { symptomology. } \\
91.5 \% \text { report less } \\
\text { unwanted side effects } \\
\text { than with prescription } \\
\text { medications. }\end{array}$ & N/A \\
\hline
\end{tabular}

1 In Canada, 1999: right to possess cannabis for medical purposes (MC); 2001: Marihuana Medical Access Regulations (MMAR) enabled individuals with the authorization of their health care practitioner to access dried MC by producing their own plants, designating someone to produce for them or purchasing Health Canada supply; 2013: Marihuana for Medical Purposes Regulations (MMPR) commercial production and distribution of MC; 2015: production and sale of cannabis oil, fresh buds and leaves; 2016: Access to Cannabis for Medical Purposes Regulations (ACMPR) set out provisions for individuals to produce a limited amount for their own medical purposes (https://www.canada.ca/en/health-canada/services/publications/drugs-health-products/understanding-new-access-to-cannabis-for-medicalpurposes-regulations.html).

2 As of May 18, 202136 states and 4 territories of the United States of America allow for the medical use of cannabis products (https://www.ncsl.org/research/health/state-medical-marijuana-laws.aspx).

3 Abbreviations: $A E$ : adverse effects; $E D$ : emergency department; $M C$ : medical cannabis/cannabis for therapeutic purpose/medical marijuana; $M M A R$ : Marihuana Medical Access Regulations; MMPR: Marihuana for Medical Purposes Regulations; NR: not reported; PTSD: Post-traumatic stress disorder; THC: delta-9-tetrahydrocannabinol; NRS: numeric rating scale; CRPS: complex regional pain syndrome

Gorter et al., 2005). Five studies received mixed funding from research grants, non-governmental organizations, dispensaries or private foundations (Nunberg et al., 2011; Piper et al., 2017; Reinarman et al., 2011; Ware et al., 2003; Bonn-Miller et al., 2014; Lintzeris et al., 2018). Those five studies also had received funding from commercial cannabis interest or cannabis patient groups (Hazekamp et al., 2013; Gorter et al., 2005; Nunberg et al., 2011; Reinarman et al., 2011; Bonn-Miller et al., 2014; Lintzeris et al., 2018).

\section{Participants' characteristics}

Participants' characteristics are described for each study in Table 1 and summarized in Table 4.

\section{Patterns of MC use}

Reported patterns of MC use for each study are presented in Table 2 and user experiences relating to the pattern or mode of use are shown in Table 3. The mode of cannabis administration was described in 36 studies. The most common form of MC consumption was inhalation (reported in 35 studies), either via smoking (joint or blunt, joint with tobacco, pipe, water pipe) or vaping (vaporizer) (Swift et al., 2005; Aggarwal et al., 2009; Hazekamp et al., 2013; Ste-Marie et al., 2016; Bottorff et al., 2011; Bruce et al., 2018; Coomber et al., 2003; Erkens et al., 2005; Haroutounian et al., 2016; Harris et al., 2000; Hoffman et al., 2017; Lucas \& Walsh, 2017; Lynch et al., 2006; Ogborne et al., 2000; Piper et al., 
2017; Reinarman et al., 2011; Schnelle et al., 1999; Sexton et al., 2016; Shiplo et al., 2016; Ste-Marie et al., 2012; Troutt \& DiDonato, 2015; Walsh et al., 2013; Ware et al., 2003; Brunt et al., 2014; Cranford et al., 2016; Crowell, 2017; Fanelli et al., 2017; Grella et al., 2014; Grotenhermen \& Schnelle, 2003; Shah et al., 2017; Zaller et al., 2015; Lavie-Ajayi \& Shvartzman, 2018; Lintzeris et al., 2018; Reiman, 2007; Sagy et al., 2019). Reported smoking prevalence ranged from 20 (Erkens et al., 2005) to 91\% (Cranford et al., 2016) and vaping prevalence from 7 (Crowell, 2017) to 53\% (Shiplo et al., 2016). Ingested (cannabis tea, baked goods, oils, tinctures, tablets and capsules) (Hazekamp et al., 2013; SteMarie et al., 2016; Bruce et al., 2018; Erkens et al., 2005; Haroutounian et al., 2016; Hoffman et al., 2017; Lucas \& Walsh, 2017; Lynch et al., 2006; Piper et al., 2017; Reinarman et al., 2011; Sexton et al., 2016; Troutt \& DiDonato, 2015; Walsh et al., 2013; Ware et al., 2003; Brunt et al., 2014; Cranford et al., 2016; Crowell, 2017; Fanelli et al., 2017; Grella et al., 2014; Grotenhermen \& Schnelle, 2003; Reiman et al., 2017; Shah et al., 2017; Zaller et al., 2015; Sagy et al., 2019) and topical administration (Ste-Marie et al., 2016; Bruce et al., 2018; Hoffman et al., 2017; Lucas \& Walsh, 2017; Sexton et al., 2016; Cranford et al., 2016) were less common forms of MC use (reported in 25 and 6 studies, respectively). The reported prevalence of ingested MC varied from 0.5 (Sexton et al., 2016) to 70\% (Erkens et al., 2005) and the prevalence of topical administration varied from 0.6 (Sexton et al., 2016) to 11\% (Cranford et al., 2016). A combined mode of cannabis consumption (e.g., both smoked MC and edible MC products) was also reported (Haroutounian et al., 2016; Shiplo et al., 2016; Ste-Marie et al., 2012; Grotenhermen \& Schnelle, 2003). Frequency and quantity of MC consumption was described in 23 (Swift et al., 2005; Aggarwal et al., 2009; Hazekamp et al., 2013; Coomber et al., 2003; Erkens et al., 2005; Harris et al., 2000; Lucas \& Walsh, 2017; Lynch et al., 2006; Ogborne et al., 2000; Reinarman et al., 2011; Sexton et al., 2016; Shiplo et al., 2016; Troutt \& DiDonato, 2015; Walsh et al., 2013; Ware et al., 2003; Bonn-Miller et al., 2014; Brunt et al., 2014; Cranford et al., 2016; Crowell, 2017; Grella et al., 2014; Shah et al., 2017; Zaller et al., 2015; Lintzeris et al., 2018) and 22 studies (Aggarwal et al., 2009; Hazekamp et al., 2013; Ste-Marie et al., 2016; Haroutounian et al., 2016; Harris et al., 2000; Lucas \& Walsh, 2017; Lynch et al., 2006; Nunberg et al., 2011; Ogborne et al., 2000; Reinarman et al., 2011; Sexton et al., 2016; Shiplo et al., 2016; Ste-Marie et al., 2012; Troutt \& DiDonato, 2015; Walsh et al., 2013; Bonn-Miller et al., 2014; Brunt et al., 2014; Cranford et al., 2016; Fanelli et al., 2017; Grotenhermen \& Schnelle, 2003; Zaller et al., 2015; Lavie-Ajayi \& Shvartzman, 2018; Sagy et al., 2019), respectively. Between 38
(Ware et al., 2003) and 90\% (Brunt et al., 2014) of participants reported daily MC consumption. Consumed quantity of MC varied from 0.05 (Fanelli et al., 2017) to 1 gram per day (Harris et al., 2000).

\section{Medical cannabis used as a substitute for prescription medications}

Of the 20 studies that examined the impact of $M C$ use on the utilization of other prescribed medications (Boehnke et al., 2016; Swift et al., 2005; Bruce et al., 2018; Haroutounian et al., 2016; Lucas \& Walsh, 2017; Lynch et al., 2006; Nunberg et al., 2011; Piper et al., 2017; Reinarman et al., 2011; Sexton et al., 2016; Troutt \& DiDonato, 2015; Corroon Jr. et al., 2017; Crowell, 2017; Grella et al., 2014; Reiman, 2009; Reiman et al., 2017; Shah et al., 2017; Webb \& Webb, 2014; Zaller et al., 2015; Lavie-Ajayi \& Shvartzman, 2018; Sagy et al., 2019), 19 reported that MC consumption was accompanied by a decrease in the number and amount of prescribed drugs used, including opioids, antidepressants, anxiolytics and benzodiazepines, and non-opioid-based pain medication (Boehnke et al., 2016; Swift et al., 2005; Bruce et al., 2018; Haroutounian et al., 2016; Lucas \& Walsh, 2017; Lynch et al., 2006; Nunberg et al., 2011; Piper et al., 2017; Reinarman et al., 2011; Sexton et al., 2016; Troutt \& DiDonato, 2015; Corroon Jr. et al., 2017; Crowell, 2017; Grella et al., 2014; Reiman, 2009; Reiman et al., 2017; Webb \& Webb, 2014; Zaller et al., 2015; Lavie-Ajayi \& Shvartzman, 2018; Sagy et al., 2019) (Table 2). In twelve studies, it had been observed that participants discontinued their use of opioids or other prescription drugs following the start of MC consumption (Swift et al., 2005; Bruce et al., 2018; Haroutounian et al., 2016; Lucas \& Walsh, 2017; Nunberg et al., 2011; Reinarman et al., 2011; Sexton et al., 2016; Corroon Jr. et al., 2017; Reiman, 2009; Webb \& Webb, 2014; Zaller et al., 2015; Lavie-Ajayi \& Shvartzman, 2018; Sagy et al., 2019), in a proportion varying from $6 \%$ (Webb \& Webb, 2014) to $63 \%$ of participants (Lucas \& Walsh, 2017). Participants also reported preferring the use of $\mathrm{MC}$ to prescription medication (Grella et al., 2014), mainly because of the adverse effects of their prescription drugs (Lynch et al., 2006).

\section{Past and current use of cannabis and other licit and illicit substances}

In 18 studies, 20 (Ste-Marie et al., 2016) to 90\% (Harris et al., 2000) of participants reported that they had previously consumed cannabis recreationally or that they consumed it simultaneously to their therapeutic cannabis use (Swift et al., 2005; Hazekamp et al., 2013; SteMarie et al., 2016; Belle-Isle et al., 2014; Degenhardt et al., 2015; Erkens et al., 2005; Harris et al., 2000; Hoffman et al., 2017; Lucas \& Walsh, 2017; Nunberg et al., 
2011; Ogborne et al., 2000; Reinarman et al., 2011; Schnelle et al., 1999; Walsh et al., 2013; Ware et al., 2003; Grella et al., 2014; Grotenhermen \& Schnelle, 2003; Shah et al., 2017; Lintzeris et al., 2018; Sagy et al., 2019) (Supplemental Table S1). One study reported that $29 \%$ of participants discovered the therapeutic effects of cannabis while using it recreationally (Swift et al., 2005). Six studies suggested that there might be a link between current $\mathrm{MC}$ use and past consumption of licit and illicit substances, as a proportion of MC users (3 to 89\%) reported a past history of substance abuse, including alcohol, cocaine, amphetamines, hallucinogens, or other prescription drugs (Perron et al., 2015; Ilgen et al., 2013; Harris et al., 2000; Bonn-Miller et al., 2014; Grella et al., 2014; Zaller et al., 2015). Moreover, some MC users considered cannabis a substitute for alcohol (up to $26 \%$ of participants) (Lucas \& Walsh, 2017) or illicit drugs (up to $16 \%$ of participants) (Zaller et al., 2015).

\section{Reported barriers to the medical use of cannabis}

Obstacles to the medical use of cannabis have been reported at several levels (Supplemental Table S2), including stigmatization from others (Ogborne et al., 2000; Piper et al., 2017), fear of discrimination (Belle-Isle et al., 2014), and physicians' unwillingness to recommend, certify, or authorize MC (Aggarwal et al., 2009; Belle-Isle et al., 2014; Lucas \& Walsh, 2017; Pedersen \& Sandberg, 2013). Some MC users expressed health concerns such as pulmonary health or fear of addiction (Swift et al., 2005; Hoffman et al., 2017; Piper et al., 2017; Grella et al., 2014), but no study explicitly investigated perceived addiction to cannabis as a treatment consequence. Difficulties in finding a consistent and affordable MC supply and fear of legal problems associated with $\mathrm{MC}$ consumption, e.g., driving after consumption, represent further obstacles to MC utilization (Swift et al., 2005; Aggarwal et al., 2009; Aggarwal et al., 2013a; Aggarwal et al., 2013b; Belle-Isle et al., 2014; Coomber et al., 2003; Hoffman et al., 2017; Lucas \& Walsh, 2017; Ogborne et al., 2000; Piper et al., 2017; Alexandre, 2011; Grotenhermen \& Schnelle, 2003; Lintzeris et al., 2018).

\section{Discussion}

\section{Main findings}

In the included studies, a majority of participants who used cannabis for therapeutic purposes to relieve pain were aged 28.4 to 62.8 years in average with a proportion of men varying between 18 and $88 \%$ (Table 4). The most frequent mode of cannabis administration was smoking. The majority of MC users consumed cannabis daily, in a quantity ranging between 0.05 and 1 gram/ day. MC users from reviewed studies reported positive effects on symptoms alleviation in addition to "secondary outcomes" such as psychological well-being.
Reported adverse effects associated with $\mathrm{MC}$ utilization were few and of minor intensity and were mainly associated with cannabis smoking, such as negative impacts on pulmonary health. MC users also reported a reduction in the use of prescription drugs for the management of chronic pain (Boehnke et al., 2016; Swift et al., 2005; Bruce et al., 2018; Haroutounian et al., 2016; Lucas \& Walsh, 2017; Lynch et al., 2006; Nunberg et al., 2011; Piper et al., 2017; Reinarman et al., 2011; Sexton et al., 2016; Troutt \& DiDonato, 2015; Corroon Jr. et al., 2017; Crowell, 2017; Grella et al., 2014; Reiman, 2009; Reiman et al., 2017; Webb \& Webb, 2014; Zaller et al., 2015; Lavie-Ajayi \& Shvartzman, 2018; Sagy et al., 2019).

\section{Strengths and limitations of the review}

To the best of our knowledge, this is the first comprehensive literature review on the perceptions of persons suffering from CMP or other chronic non-cancer pain, who used cannabis for therapeutic reasons. The information gathered in this review represents an opportunity to better understand the perspective of different types of $\mathrm{MC}$ users on the multiple dimensions of its consumption, in particular barriers, advantages, and drawbacks.

However, this review has several limitations, related principally to methodological weaknesses in an important proportion of the included studies.

\section{Selection and recruitment of participants}

For $41 \%$ of participants, they have been recruited at MC dispensaries, MC associations, or MC advocacy groups, including four studies performed in countries without a legal framework for access to MC (Swift et al., 2005; Coomber et al., 2003; Lintzeris et al., 2018; Pedersen et al., 2016). This might have introduced selection and information biases, as it has been reported that people who are already familiar with cannabis through recreational use, may use cannabis for medical reasons (Bigand et al., 2019; Lum et al., 2019). Indeed, among the about 30\% of studies reporting on prior cannabis use, many $\mathrm{MC}$ users reported recreational cannabis use prior or simultaneously to MC use. Some MC users reported that it was during the recreational use of cannabis that they discovered its therapeutic effects. Moreover, people who are attending these centers may not use cannabis exclusively for medical reasons. In addition, MC users who had stopped MC consumption participated only marginally in these studies. Prevalence of adverse effects might therefore be underestimated. Furthermore, a subgroup of those studies, for which the source of funding was reported, was financially supported by cannabis interest or patient groups. This may have introduced a positive bias toward the use of cannabis against chronic pain. Therefore, we can argue that 
Table 2 Patterns of medical cannabis use and utilization of medical cannabis as a substitute for prescription medications

\begin{tabular}{|c|c|c|c|c|c|}
\hline Article & $\begin{array}{l}\text { Mode of cannabis } \\
\text { administration }\end{array}$ & Mode advantages & $\begin{array}{l}\text { Mode } \\
\text { Disadvantages }\end{array}$ & $\begin{array}{l}\text { Quantity } 1 / \text { Frequency } \\
\text { of cannabis use }\end{array}$ & $\begin{array}{l}\text { Cannabis used as a } \\
\text { substitute for } \\
\text { prescription medications }\end{array}$ \\
\hline $\begin{array}{l}\text { Aggarwal et al. } \\
2009\end{array}$ & $\begin{array}{l}\text { When mentioned, mainly } \\
\text { smoking. }\end{array}$ & Not reported & Not reported & $\begin{array}{l}\text { From "as needed" to over } \\
10 \text { times daily. } \\
\text { From } 1 / 2 \text { to } 14 \mathrm{~g} / \text { week. }\end{array}$ & Not reported \\
\hline $\begin{array}{l}\text { Boehnke et al. } \\
2016\end{array}$ & Not reported & Not reported & Not reported & Not reported & $\begin{array}{l}45 \% \text { of respondents reported } \\
\text { a } 64 \% \text { reduction in opioid } \\
\text { use with medical cannabis } \\
\text { use. } \\
\text { Decrease in the number of } \\
\text { medications classes used with } \\
\text { medical cannabis use }(2.38 \text { to } \\
1.81, P<.001) \text {. }\end{array}$ \\
\hline $\begin{array}{l}\text { Bonn-Miller } \\
\text { et al. } 2014\end{array}$ & Not reported & Not reported & Not reported & $\begin{array}{l}\text { Participants used } 2 \text { to } 3 \\
\text { times/day. } \\
\text { They used 6-12 g/week. }\end{array}$ & Not reported \\
\hline $\begin{array}{l}\text { Bottorff et al. } \\
2011\end{array}$ & Primarily smoking. & $\begin{array}{l}\text { Smoking: } \\
\text { - convenient } \\
\text { - affordable } \\
\text { - more effective } \\
\text { regulation of } \\
\text { dosing. }\end{array}$ & $\begin{array}{l}\text { Smoking- } \\
\text { related } \\
\text { concerns: } \\
\text { - coughing } \\
\text { - breathing } \\
\text { difficulties } \\
\text { - fear of lung } \\
\text { cancer. }\end{array}$ & Not reported & Not reported \\
\hline $\begin{array}{l}\text { Bruce et al. } \\
2018\end{array}$ & $\begin{array}{l}60 \% \text { of participants preferred } \\
\text { smoking; } \\
20 \% \text { vaporizing; } \\
17 \% \text { ingestion; } \\
3 \% \text { topical use. }\end{array}$ & Not reported & Not reported & Not reported & $\begin{array}{l}\text { medical cannabis use } \\
\text { reported as: } \\
\text { - alternative to prescription } \\
\text { (opioids, anticonvulsants, } \\
\text { anti-inflammatories) or OTC } \\
\text { medications; } \\
\text { - complementary, with } \\
\text { prescription medications; } \\
\text { - a means for tapering off } \\
\text { prescription medications. }\end{array}$ \\
\hline $\begin{array}{l}\text { Brunt et al. } \\
2014\end{array}$ & $81 \%$ inhalation; $19 \%$ tea. & Not reported & Not reported & $\begin{array}{l}90 \% \text { of participants used } \\
\text { daily. } \\
\text { Mean cumulative dose: } \\
0.65 \pm 0.63 \mathrm{~g} / \text { day }[4.5 \mathrm{~g} / \\
\text { week]. }\end{array}$ & Not reported \\
\hline $\begin{array}{l}\text { Coomber et al. } \\
2003\end{array}$ & 73\% smoking. & $\begin{array}{l}\text { Smoking: less } \\
\text { amount required } \\
\text { than eating or } \\
\text { drinking. }\end{array}$ & Not reported & $\begin{array}{l}48 \% \text { used daily; } 24 \% \text { used } \\
1-3 \text { times/week. } \\
24 \% \text { used } 1-3 \text { joints/day. }\end{array}$ & Not reported \\
\hline $\begin{array}{l}\text { Corroon et al. } \\
2017\end{array}$ & Not reported & Not reported & Not reported & Not reported & $\begin{array}{l}\text { Odds of reporting } \\
\text { substitution } 4.59 \text { ( } 95 \% \mathrm{Cl} \text {, } \\
\text { 3.87-5.43) times greater } \\
\text { among self-identified medical } \\
\text { cannabis than among non- } \\
\text { medical cannabis users. } \\
\text { Most common classes of } \\
\text { drugs substituted: narcotics/ } \\
\text { opioids (36\%), anxiolytics/BZD } \\
\text { (14\%), and antidepressants } \\
(13 \%) \text {. }\end{array}$ \\
\hline $\begin{array}{l}\text { Cranford et al. } \\
2016\end{array}$ & $\begin{array}{l}91 \% \text { reported smoking; } \\
44 \% \text { eating, drinking, or } \\
\text { ingesting; } \\
39 \% \text { vaping; } \\
11 \% \text { topical use. }\end{array}$ & Not reported & Not reported & $\begin{array}{l}74 \% \text { of participants used } \\
\text { almost daily. } \\
\text { From none to }>1 \text { ounce } \\
\text { ( } 14.5 \% \text { of participants)/ } \\
\text { month }[0 \text { to } 6.5 \mathrm{~g} / \text { week]. }\end{array}$ & Not reported \\
\hline
\end{tabular}


Table 2 Patterns of medical cannabis use and utilization of medical cannabis as a substitute for prescription medications (Continued)

\begin{tabular}{|c|c|c|c|c|c|}
\hline Article & $\begin{array}{l}\text { Mode of cannabis } \\
\text { administration }\end{array}$ & Mode advantages & $\begin{array}{l}\text { Mode } \\
\text { Disadvantages }\end{array}$ & $\begin{array}{l}\text { Quantity }{ }^{1} / \text { Frequency } \\
\text { of cannabis use }\end{array}$ & $\begin{array}{l}\text { Cannabis used as a } \\
\text { substitute for } \\
\text { prescription medications }\end{array}$ \\
\hline Crowell 2017 & $\begin{array}{l}\text { Most frequent mode: } 80 \% \\
\text { smoking; } 7 \% \text { vaporizing; } 12 \% \\
\text { edibles. }\end{array}$ & Not reported & Not reported & $\begin{array}{l}3-4 \text { times/day (41.6-37.9\%); } \\
1-2 \text { times/day (38.7-27.1\%). }\end{array}$ & $\begin{array}{l}\text { At first visit: } 50 \% \text { of } \\
\text { participants had reduced use } \\
\text { of pain medication; at visit 2: } \\
62.4 \% \text {; at visit 3: } 60 \% \text {. }\end{array}$ \\
\hline $\begin{array}{l}\text { Erkens et al. } \\
2005\end{array}$ & $70 \%$ as tea; $20 \%$ smoked. & Not reported & Not reported & 1 to 4 times/day. & Not reported \\
\hline $\begin{array}{l}\text { Fanelli et al. } \\
2017\end{array}$ & $\begin{array}{l}\text { Primarily cannabis tea } \\
\text { (smoking cannabis not } \\
\text { permitted in Italy). } \\
92 \% \text { used } 22 \% \mathrm{THC} /<1 \% \text { CBD } \\
\text { Bedrocan. }\end{array}$ & Not reported & Not reported & $\begin{array}{l}\text { From } 56.7 \pm 45.5 \mathrm{mg} / \text { day } \\
{[0.4 \mathrm{~g} / \text { week] at treatment }} \\
\text { initiation; to } 67.0 \pm 58.8 \\
\mathrm{mg} / \text { day }[0.5 \mathrm{~g} / \text { week] at } \\
\text { follow-up ( } 98 \pm 145 \text { days). }\end{array}$ & Not reported \\
\hline $\begin{array}{l}\text { Grella et al. } \\
2014\end{array}$ & $\begin{array}{l}51 \% \text { used a pipe/water pipe, } \\
47 \% \text { smoked joints or blunts; } \\
23 \% \text { used vaporizers; } 16.5 \% \\
\text { edibles; } 3.3 \% \text { oral tincture. }\end{array}$ & Not reported & Not reported & $\begin{array}{l}2.5 \pm 2.6 \text { dispensary visits/ } \\
\text { month. } \\
57 \% \text { of focus group } \\
\text { participants used several } \\
\text { times daily. }\end{array}$ & $\begin{array}{l}\text { A common theme among } \\
\text { participants was the } \\
\text { preference for using medical } \\
\text { cannabis instead of } \\
\text { prescription medications. } \\
\text { In the previous } 30 \text { days, } 7 \% \\
\text { had non-medical use of pain- } \\
\text { killers, } 4 \% \text { of stimulants, and } \\
8 \% \text { of tranquillizers. }\end{array}$ \\
\hline $\begin{array}{l}\text { Grotenhermen } \\
\text { \& Schnelle } \\
2003\end{array}$ & $\begin{array}{l}56 \% \text { inhalation; } 17 \% \text { oral use; } \\
23 \% \text { used both modes. }\end{array}$ & Not reported & Not reported & $\begin{array}{l}\text { Average doses of natural } \\
\text { cannabis products ( } 109 \\
\text { participants): } 1.3 \pm 0.9 \\
\text { (0.02-3.5) g/day }[9.1 \mathrm{~g} / \\
\text { week]. }\end{array}$ & Not reported \\
\hline $\begin{array}{l}\text { Haroutounian } \\
\text { et al. } 2016\end{array}$ & $\begin{array}{l}77 \% \text { received cannabis } \\
\text { cigarettes; } 5 \% \text { received a } \\
\text { combination of cigarettes and } \\
\text { drops; } 10 \% \text { only drops; } 5 \% \text { only } \\
\text { cookies; } 3 \% \text { combination of } \\
\text { cookies and drops. }\end{array}$ & Not reported & Not reported & $\begin{array}{l}\text { Monthly prescribed } \\
\text { cannabis: } 43.2 \pm 17.9 \mathrm{~g} / \\
\text { month }\end{array}$ & $\begin{array}{l}44 \% \text { of participants on opioid } \\
\text { therapy at baseline had } \\
\text { discontinued }(P<0.001) \text {. }\end{array}$ \\
\hline $\begin{array}{l}\text { Harris et al. } \\
2000\end{array}$ & Mainly smoking. & Not reported & Not reported & $\begin{array}{l}65 \% \text { daily use. } \\
86 \% \text { used } \geq 2 \text { cigarettes/ } \\
\text { day. } \\
1 \text { g/day. }\end{array}$ & Not reported \\
\hline $\begin{array}{l}\text { Hazekamp } \\
\text { et al. } 2013\end{array}$ & $\begin{array}{l}63 \% \text { preferred smoking; } 24 \% \\
\text { vaporizing; } 8 \% \text { food/tincture; } \\
2.4 \% \text { tea. } \\
\text { Fewer participants had } \\
\text { experience with dronabinol } \\
11.3 \% \text {, nabilone } 2.1 \% \text {, } \\
\text { nabiximols } 1.1 \% \text {. }\end{array}$ & Not reported & Not reported & $\begin{array}{l}\text { On average } \\
\text { Times per day: smoking 6.0, } \\
\text { vaporizing 5.2, tea 1.9, } \\
\text { food/tincture } 1.8 \text {. } \\
\text { Grams per day: smoking 3, } \\
\text { vaporizing 3, tea 2.4, food/ } \\
\text { tincture } 3.4 \mathrm{~g} \text {. }\end{array}$ & Not reported \\
\hline $\begin{array}{l}\text { Hoffman et al. } \\
2017\end{array}$ & $\begin{array}{l}73 \% \text { smoking; } 32 \% \text { ingestion; } \\
23 \% \text { vaporizing; } 9 \% \text { topical } \\
\text { use. }\end{array}$ & $\begin{array}{l}\text { - Most felt vaporizing } \\
\text { healthier than } \\
\text { smoking. } \\
\text { - Of those who } \\
\text { ingested, most felt } \\
\text { it more effective for } \\
\text { pain relief than } \\
\text { smoking. }\end{array}$ & Not reported & Not reported & Not reported \\
\hline $\begin{array}{l}\text { Lavie-Ajayi and } \\
\text { Shvartzman } \\
2018\end{array}$ & $\begin{array}{l}\text { Smoking and others (not } \\
\text { reported) }\end{array}$ & Not reported & $\begin{array}{l}\text { Unpleasant } \\
\text { taste or smell } \\
\text { of cannabis. }\end{array}$ & $20-60 \mathrm{~g} / \mathrm{month}$ & $\begin{array}{l}\text { Reduction in side effects of } \\
\text { prescription medication. } \\
\text { Medical cannabis use } \\
\text { reported as alternative to } \\
\text { other medication used for } \\
\text { sleeplessness, irritability, } \\
\text { restlessness, inability to focus, } \\
\text { and depression. }\end{array}$ \\
\hline $\begin{array}{l}\text { Lintzeris et al. } \\
2018\end{array}$ & Inhalation (83.4\%) & Not reported & Not reported & $\begin{array}{l}\text { Participants used } 3 \text { times/ } \\
\text { day }\end{array}$ & Not reported \\
\hline
\end{tabular}


Table 2 Patterns of medical cannabis use and utilization of medical cannabis as a substitute for prescription medications (Continued)

\begin{tabular}{|c|c|c|c|c|c|}
\hline Article & $\begin{array}{l}\text { Mode of cannabis } \\
\text { administration }\end{array}$ & Mode advantages & $\begin{array}{l}\text { Mode } \\
\text { Disadvantages }\end{array}$ & $\begin{array}{l}\text { Quantity } 1 / \text { Frequency } \\
\text { of cannabis use }\end{array}$ & $\begin{array}{l}\text { Cannabis used as a } \\
\text { substitute for } \\
\text { prescription medications }\end{array}$ \\
\hline $\begin{array}{l}\text { Lucas and } \\
\text { Walsh } 2017\end{array}$ & $\begin{array}{l}90 \% \text { had tried joints, } 86 \% \\
\text { vaporizers, } 76 \% \text { oral/edibles, } \\
16 \% \text { topical. } \\
\text { Primary methods of use: } \\
38 \% \text { vaporizing, } 25 \% \text { smoking } \\
\text { joints, } 14 \% \text { oral/edibles, } 12 \% \\
\text { waterpipe/bongs, } 11 \% \text { pipes, } \\
1 \% \text { topicals. } \\
\text { Preferred method: } \\
44 \% \text { vaporization, } 23 \% \text { edibles. }\end{array}$ & Not reported & Not reported & $\begin{array}{l}88 \% \text { of participants used at } \\
\text { least daily. } \\
\text { Modal: } 1-2 \mathrm{~g} / \text { day }[7-14 \mathrm{~g} / \\
\text { week], with } 29 \%(\mathrm{n}=79) \\
\text { using a larger amount. }\end{array}$ & $\begin{array}{l}63 \% \text { of participants reported } \\
\text { substitution for prescription } \\
\text { medication. } \\
\text { The most common form of } \\
\text { substitution was for opioids } \\
\text { (32\%), BZD (16\%), and } \\
\text { antidepressants (12\%). }\end{array}$ \\
\hline $\begin{array}{l}\text { Lynch et al. } \\
2006\end{array}$ & $\begin{array}{l}\text { All participants reported } \\
\text { smoking some of the time. } \\
30 \% \text { used both the smoking } \\
\text { and oral routes; } \\
7 \% \text { used primarily the oral } \\
\text { route. }\end{array}$ & Not reported & Not reported & $\begin{array}{l}1 \text { to }>6 \text { times/day. } \\
2.5 \mathrm{~g} / \text { day [17.5g/week]. }\end{array}$ & $\begin{array}{l}70 \% \text { decreased use of other } \\
\text { medications that had been } \\
\text { causing side effects (NSAIDs, } \\
\text { opioids, and antidepressants). }\end{array}$ \\
\hline $\begin{array}{l}\text { Nunberg et al. } \\
2011 \text { \& } \\
\text { Reinarman } \\
\text { et al. } 2011\end{array}$ & Not reported & Not reported & Not reported & Not reported & $\begin{array}{l}51 \% \text { reported using cannabis } \\
\text { as a substitute for } \\
\text { prescription medications. }\end{array}$ \\
\hline $\begin{array}{l}\text { Ogborne et al. } \\
2000\end{array}$ & Mainly smoking. & $\begin{array}{l}\text { Smoking: } \\
\text { - enjoyable } \\
\text { - immediate, } \\
\text { effective } \\
\text { - less expensive } \\
\text { Eating/drinking: } \\
\text { - "less of a head } \\
\text { stone..." } \\
\text { - longer lasting } \\
\text { - no smell }\end{array}$ & $\begin{array}{l}\text { Smoking: } \\
\text { - Respiratory } \\
\text { side effects } \\
\text { (cough, throat } \\
\text { irritation) } \\
\text { Eating/drinking: } \\
\text { - too slow } \\
\text { - less effective } \\
\text { - more difficult } \\
\text { to regulate in } \\
\text { terms of dose. }\end{array}$ & $\begin{array}{l}70 \% \text { of participants } \\
\text { smoked every day. } \\
\text { They smoked } 1 \text { to } 10 \\
\text { joints/day. } \\
\text { They used } 28 \text { to } 56 \mathrm{~g} / \\
\text { month }[6.5-13 \mathrm{~g} / \text { week]. }\end{array}$ & Not reported \\
\hline $\begin{array}{l}\text { Piper et al. } \\
2017\end{array}$ & $\begin{array}{l}46 \% \text { of participants smoked } \\
\text { medical cannabis; } 23 \% \\
\text { vaporizing; } 14 \% \text { edibles; } 12 \% \\
\text { tincture. }\end{array}$ & $\begin{array}{l}\text { Vaping: medical } \\
\text { cannabis } \\
\text { administered with } \\
\text { joints was } \\
\text { significantly more } \\
\text { expensive than via } \\
\text { vaporizer. }\end{array}$ & $\begin{array}{l}\text { Smoking: } \\
\text { - not always } \\
\text { convenient } \\
\text { - gross } \\
\text { - bad taste. } \\
\text { Vaporizing: } \\
\text { - cumbersome } \\
\text { - too } \\
\text { expensive. } \\
\text { Edibles: } \\
\text { - Lack of } \\
\text { availability. } \\
\text { Tincture: } \\
\text { - takes too } \\
\text { long } \\
\text { - complex } \\
\text { dosing. }\end{array}$ & Not reported & $\begin{array}{l}\text { Decrease in prescription } \\
\text { medications. }\end{array}$ \\
\hline Reiman 2009 & Not reported & Not reported & Not reported & Not reported & $\begin{array}{l}66 \% \text { of participants reported } \\
\text { having used cannabis as a } \\
\text { substitute for prescription } \\
\text { drugs. }\end{array}$ \\
\hline $\begin{array}{l}\text { Reiman et al. } \\
2017\end{array}$ & $\begin{array}{l}50 \% \text { smoking; } 30 \% \text { vaporizing; } \\
10 \% \text { edibles. }\end{array}$ & Not reported & Not reported & Not reported & $\begin{array}{l}97 \% \text { of participants decreased } \\
\text { the amount of opioids they } \\
\text { consume when they also use } \\
\text { cannabis. } \\
96 \% \text { do not need to take as } \\
\text { much of their nonopioid- } \\
\text { based pain medication when }\end{array}$ \\
\hline
\end{tabular}


Table 2 Patterns of medical cannabis use and utilization of medical cannabis as a substitute for prescription medications (Continued)

\begin{tabular}{llll}
\hline Article & $\begin{array}{l}\text { Mode of cannabis } \\
\text { administration }\end{array}$ & Mode advantages & $\begin{array}{l}\text { Mode } \\
\text { Disadvantages of cannabis use }\end{array}$
\end{tabular}

Cannabis used as a
substitute for
prescription medications

\begin{tabular}{|c|c|}
\hline $\begin{array}{l}\text { Reinarman } \\
\text { et al. } 2011\end{array}$ & $\begin{array}{l}86 \% \text { smoking; } 24 \% \text { orally; } 22 \% \\
\text { vaporizing. }\end{array}$ \\
\hline $\begin{array}{l}\text { Sagy et al. } \\
2019\end{array}$ & Smoking, oil \\
\hline $\begin{array}{l}\text { Schnelle et al. } \\
1999\end{array}$ & $\begin{array}{l}49 \% \text { inhalation; } 14 \% \text { eating, } \\
\text { drinking; } 36 \% \text { used both the } \\
\text { oral and inhalation routes } \\
4 \% \text { used dronabinol. }\end{array}$ \\
\hline $\begin{array}{l}\text { Sexton et al. } \\
2016\end{array}$ & $\begin{array}{l}84 \% \text { inhalation: } 32 \% \text { pipes, } \\
19 \% \text { bongs, } 16.5 \% \text { joints/ } \\
\text { blunts, 16\% vaporizer; } 8 \% \text { oral } \\
\text { (edibles, tinctures, capsules); } \\
6 \% \text { concentrates (oil, keif, } \\
\text { hash); } 0.6 \% \text { topical; } 0.5 \% \text { fresh } \\
\text { juice. }\end{array}$ \\
\hline
\end{tabular}

Not reported

Not reported

Not reported

Not reported

Not reported

Not reported

$67 \%$ daily use; $53 \%$ use $1-2$ times per day. $\leq 3$ grams (40\%) to $\geq 7$ grams (23\%) per week.

From 670 to 1000 mg/day

Not reported Not reported

Not reported

$61 \%$ reported using 1-5

they use cannabis.

$50.9 \%$ of participants reported use of medical cannabis to substitute prescription medication.

After 6 months of medical cannabis therapy, a substantial proportion of participants stopped or decreased the dosage of other medical therapies.

Not reported hits per smoking session, $21.3 \%$ reported $6-10$ hits, $18 \%$ reported $>10$ hits/ session.

$25 \%$ reported using less than 1 once/day; 1-4 times/day (47.6\%); 5-10 times/day (14.9\%), and $12.2 \%$ reported using all day, every day. Reported use (g/week): < $1(12.3 \%) ; 1-2(20.3 \%) ; 3-$ $5(31.8 \%) ; 7(26.1 \%) ; 28$ $(6 \%),>28(3.4 \%)$.

Shah et al. 2017

54\% smoking; 29\% tablets; $8 \%$ Not reported edibles.

Not reported

$62.5 \%$ of medical cannabis users endorsed daily use, $21 \%$ weekly use.

- Harm from

Almost every day: $77 \%,>$ smoking. once a day: $82 \%$. vapourizing. $47 \%$ smoking a joint. Among those reporting multiple modes: $25 \%$ eating in food, $23 \%$ drinking.

Ste-Marie et al. Out of the 59 medical $2012 \quad$ cannabis users: $80 \%$ smoked herbal cannabis; $24 \%$ used prescription cannabinoids; $3 \%$ used both.

Ste-Marie et al. $86 \%$ smoking; 21\% vaporizing; Not reported 2016 $18 \%$ ingestion; $4 \%$ topical.

Time to onset of

effect.

- Ability to find

correct dose.

- Smoking lower cost and more

accessible.

- Eating/drinking had more durable effect.

Not reported

Eating in foods: $1.8 \pm 1.6 \mathrm{~g} /$ day $[12.6 \mathrm{~g} /$

- producing the week].

worst high

- most stigma

- hardest to

find a correct

dose.

Not reported

$$
\text { g/week]. }
$$

Not reported

For the 22 patients who recorded amounts used, most reported $\leq 1.5 \mathrm{~g} /$ day [ $10.5 \mathrm{~g} /$ week $]$.

Swift et al.

91\% smoked.

$74 \%$ considered smoking the most helpful route.
Smoking:

- Instant effect.

- Ease of titration.

- Cost-effectiveness.

Edibles:

- Healthier

- Tasty when cooked
Smoking:

- Detrimental

$75 \%$ used at least weekly,

$59 \%$ used almost daily,

to respiratory $22 \%$ used "as required."

function (and

health)

Edibles:

- Availability of
$60 \%$ reported substitute medical cannabis for prescription drugs, 25\% for pain medications, including opiates.

Cannabis use was not associated with a significantly lower morphine equivalence level for participants using prescription opioids.

Not reported

Not reported

$62 \%$ of participants claimed they decreased or discontinued their use of other medicines with medical cannabis use. 
Table 2 Patterns of medical cannabis use and utilization of medical cannabis as a substitute for prescription medications (Continued)

\begin{tabular}{|c|c|c|c|c|c|}
\hline Article & $\begin{array}{l}\text { Mode of cannabis } \\
\text { administration }\end{array}$ & Mode advantages & $\begin{array}{l}\text { Mode } \\
\text { Disadvantages }\end{array}$ & $\begin{array}{l}\text { Quantity } 1 / \text { Frequency } \\
\text { of cannabis use }\end{array}$ & $\begin{array}{l}\text { Cannabis used as a } \\
\text { substitute for } \\
\text { prescription medications }\end{array}$ \\
\hline & & $\begin{array}{l}\text { in a recipe } \\
\text { - Less obvious } \\
\text { - Slow onset and } \\
\text { long-lasting effects. }\end{array}$ & $\begin{array}{l}\text { recipes } \\
\text { - Difficulties } \\
\text { with titration } \\
\text { - expensive and } \\
\text { ineffective for } \\
\text { rapid relief. }\end{array}$ & & \\
\hline $\begin{array}{l}\text { Troutt \& } \\
\text { DiDonato } 2015\end{array}$ & $\begin{array}{l}67 \% \text { inhalation: } \sim 42 \% \\
\text { smoking, } \sim 25 \% \text { vaporizing; } \sim \\
27 \% \text { edibles; } \sim 4 \% \text { tincture; } \sim \\
2 \% \text { oils. }\end{array}$ & Not reported & Not reported & $\begin{array}{l}84 \% \text { used several times per } \\
\text { week or more, } 61 \% \text { used } \\
\text { daily. } \\
78 \% \text { used }<14 \mathrm{~g} / \text { month } \\
{[3.2 \mathrm{~g} / \text { week]. }}\end{array}$ & $\begin{array}{l}90 \% \text { of chronic pain, } 81 \% \text { of } \\
\text { arthritis, } 94 \% \text { of fibromyalgia, } \\
\text { and } 84 \% \text { of osteoarthritis } \\
\text { patients report less frequent } \\
\text { use of other medications. }\end{array}$ \\
\hline $\begin{array}{l}\text { Walsh et al. } \\
2013\end{array}$ & $\begin{array}{l}57 \% \text { smoking; } 43 \% \text { vaporizing; } \\
28 \% \text { orally. }\end{array}$ & Not reported & Not reported & $\begin{array}{l}53 \% \text { used } 2-3 \text { times/day, } \\
42 \text { used } \geq 4 \text { times/day. } \\
45 \% \text { used }>14 \text { g/week. }\end{array}$ & \\
\hline $\begin{array}{l}\text { Ware et al. } \\
2003\end{array}$ & $\begin{array}{l}\text { Among users for pain: } \\
81 \% \text { joint, } 47 \% \text { joint with } \\
\text { tobacco, } 34 \% \text { pipe, } 16 \% \text { water } \\
\text { pipe; } 9 \% \text { orally. }\end{array}$ & Not reported & Not reported & $\begin{array}{l}53 \% \text { used } \leq 4 \text { puffs/dosing } \\
\text { interval, } 25 \% \text { smoked a } \\
\text { whole cannabis cigarette, } \\
12 \% \text { smoked } \geq 1 \text { joint. } \\
22 \% \text { of these participants } \\
\text { used cannabis }>1 \text { time/ } \\
\text { day, } 16 \% \text { used daily, } 25 \% \\
\text { used weekly and } 28 \% \\
\text { rarely used medical } \\
\text { cannabis. }\end{array}$ & Not reported \\
\hline $\begin{array}{l}\text { Webb \& Webb } \\
2014\end{array}$ & Not reported & Not reported & Not reported & Not reported & $\begin{array}{l}6 \% \text { wrote brief notes relating } \\
\text { how cannabis helped them } \\
\text { to decrease or to discontinue } \\
\text { other medications. }\end{array}$ \\
\hline $\begin{array}{l}\text { Zaller et al. } \\
2015\end{array}$ & $\begin{array}{l}74 \% \text { smoking; } 16.5 \% \\
\text { vaporizing; } 7 \% \text { orally. }\end{array}$ & Not reported & Not reported & $\begin{array}{l}60.5 \% \text { used } \geq 3 \text { times/day. } \\
48.5 \% \text { used } 3-8 \mathrm{~g} / \text { day, } \\
34.5 \% \text { used }>8 \text { g/day [ } 21- \\
56 \text { g/week]. }\end{array}$ & $\begin{array}{l}55 \% \text { indicated they had used } \\
\text { cannabis as a substitute for } \\
\text { prescription medications. }\end{array}$ \\
\hline
\end{tabular}

study participants were likely not representative of the general population with CMP or other chronic non-cancer pain, since a relevant subgroup of persons suffering from CMP or chronic non-cancer pain, but not considering $\mathrm{MC}$ as a therapeutic option, are not represented in the included studies. For instance, the mean age of MC users in the included studies (28.462.8 years) was lower than that of patients suffering from CMP, the incidence of which increases with age (Yelin et al., 2016). In addition, overall, the proportion of men in the included studies was higher than that of women, although CMP affects more often women than men (Yelin et al., 2016), suggesting a possible "gender effect": with cannabis consumption being more popular among men than women (Carliner et al., 2017) and considering that individuals who already have consumed cannabis seem to be more disposed to use it as a therapeutic agent, it is possible that men are more likely to use cannabis for therapeutic purpose than women (Swift et al., 2005).
Use of MC with a medical prescription, in a dispensary or in self-medication

In addition, in many studies, it was difficult to distinguish between qualified and self-identified MC users, as it was not specified whether $\mathrm{MC}$ use was endorsed by a physician-confirmed diagnosis. It was impossible to estimate the prevalence of each type of user in all selected studies and it was thus not possible to estimate the overall prevalence of self-medication in these studies. Prevalence of self-medication is an important aspect, as it is increasing (Park \& Wu, 2017), but self-identified MC users may have different characteristics than qualified $\mathrm{MC}$ users. It may become important for physicians to consider the possibility of self-medication with cannabis among their patients with CMP or other chronic pain.

\section{Other methodological concerns}

The included studies also varied greatly in terms of objectives, methodology, and participants' populations, 
Table 3 Perceived positive and negative effects

\begin{tabular}{lll}
\hline & Perceived positive effect & Perceived negative effect \\
\hline Smoking medical cannabis & Enjoyable & Respiratory side effect \\
& Easy to titrate & Bad smell \\
& Immediate pain relief & Bad taste \\
Ldible medical cannabis & Hexpensive compared to edible or vaping cannabis & \\
& Tasty when cooked in a recipe & Long-lasting pain relief \\
No specific medical cannabis & Alleviation of pain, headache, anxiety & Increase of appetite \\
use & Positive effect on mood & Drowsiness \\
& Improvement of their general quality of life & Cognitive effects \\
& Longer effect and less adverse effect compare to opioids and other prescription & Respiratory effects (for cannabis \\
& smodication & \\
\hline
\end{tabular}

with 13 studies out of 49 (27\%) having less than 100 participants. Data obtained during interviews or from questionnaires were self-reported and may suffer from recall or social desirability bias, while chart reviews may not have allowed to capture patient perceptions. The different legal frameworks regarding MC use across the different countries and periods of time might have influenced the availability and quality of
$M C$, the sample size of the studies, and the availability of information on MC users. The conditions permitting to be registered as a MC user as well as access to MC vary between countries, states, and over time. For example, MC can be obtained from pharmacies in the Netherlands (Erkens et al., 2005; Brunt et al., 2014; Hazekamp \& Heerdink, 2013), from special dispensaries in some states of the USA (Aggarwal

Table 4 Patients' characteristics

\begin{tabular}{|c|c|c|c|c|}
\hline $\begin{array}{l}\text { Age of } \\
\text { participants }\end{array}$ & $\begin{array}{l}\text { Mean age: } 28 \text { to } 61 \text { years. } \\
\text { Interval } 14-93 \text { years }\end{array}$ & & & \\
\hline $\begin{array}{l}\text { Proportion of } \\
\text { women in the } \\
\text { studies }\end{array}$ & 12 to $82 \%$ & & & \\
\hline \multirow[t]{2}{*}{$\begin{array}{l}\text { Type of MC } \\
\text { user in the } \\
\text { studies }\end{array}$} & 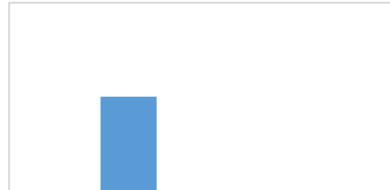 & & & \\
\hline & $\begin{array}{l}\text { Qualified MC Self-identified } \\
\text { user (legally MC users } \\
\text { authorized) }\end{array}$ & $\begin{array}{l}\text { Self-identified } \\
\text { and qualified } \\
\text { MC user }\end{array}$ & $\begin{array}{l}\text { User seeking } \\
\text { MC } \\
\text { certification } \\
\text { or } \\
\text { recertification }\end{array}$ & $\begin{array}{c}\text { Insufficient } \\
\text { details to } \\
\text { discriminate } \\
\text { between the } \\
\text { two } \\
\text { categories }\end{array}$ \\
\hline
\end{tabular}


et al., 2009; Aggarwal et al., 2013a; Piper et al., 2017; Troutt \& DiDonato, 2015; Bonn-Miller et al., 2014; Grella et al., 2014; Zaller et al., 2015), and since 2013 from registered producers in Canada (Ste-Marie et al., 2016; Lucas \& Walsh, 2017; Shiplo et al., 2016), as reflected in the included studies with participants recruited at dispensaries, registration clinics, or through online advertisement.

\section{Chronic musculoskeletal pain}

Although our scoping review aimed to report on $\mathrm{MC}$ users dealing with CMP, we identified only two studies that specifically assessed this type of chronic pain (SteMarie et al., 2016). The remaining studies comprised various proportions of participants suffering from CMP or non-specified chronic non-cancer pain. This heterogeneity among $\mathrm{MC}$ users may have influenced the reported information on MC consumption and its effects, since no distinction has been made relative to participants' disease. Considering that the pathophysiology of pain varies depending on the syndrome (McMahon et al., 2013), clinical characteristics of participants should be as homogeneous as possible in order to conclude on the effects of MC on participants' pain perception. It is thus somewhat reassuring that the two articles reporting specifically on patients suffering from CMP observed similar results as the other studies reporting on more heterogeneous populations. Indeed, among 1000 consecutive rheumatology patients, Ste-Marie et al. observed that 28 patients consumed MC. In agreement with the other studies, the authors observed that MC users were younger than the other patients of this clinic (52.8 vs. 62.8 years) and were more likely to be male $(P=0.051)$. In addition, $\mathrm{MC}$ users had previously consumed cannabis recreationally and $39.3 \%$ of the MC users reported to consume cannabis recreationally, in addition to $\mathrm{MC}$ (Ste-Marie et al., 2016).

\section{Gaps in the literature}

We identified some gaps in the literature that need to be addressed to better understand patients' utilization of MC against MCP and unspecified chronic non-cancer pain. First, future studies should include participants who have stopped MC consumption or do not want to consider it, in order to understand the reasons that lead to discontinuation or rejection of $\mathrm{MC}$, such as stigmatization of cannabis users or onset of adverse effects associated with $\mathrm{MC}$ use. As an example, Zolotov et al. reported that among participants who consumed cannabis for medical reasons, including chronic noncancer pain (47.5\%), those who abandoned MC (20\%) experienced more frequent adverse effects (dizziness, dehydrated mouth, fatigue, mild anxiety, and feeling "weird") than those who continued MC use $(P<0.05)$ (Zolotov et al., 2016).

Supported by a recent literature review, it would be interesting to better understand the point of view of physicians to identify the major factors which impact the decision of prescribing or not medical cannabis for patients who suffered from chronic pain (Gardiner et al., 2019). This would bring new knowledge on whether prescribers need support during the informed decisionmaking regarding the use of $\mathrm{MC}$ to treat $\mathrm{CMP}$. The debate among physicians whether or not to prescribe $\mathrm{MC}$ is ongoing and has recently been presented in the literature (Caulley et al., 2018). Moreover, a changing legal framework for recreational cannabis may influence the perception of physicians regarding treatment with MC.

The use of MC as a substitute for other drugs, including opioids and other prescription medications, will need to be investigated for improved decisionmaking regarding $\mathrm{MC}$ prescribing, since opioids present serious, well-documented adverse effects. Currently, clinical guidelines in some countries, e.g., Canada, only support the use of MC for specific medical conditions, including neuropathic pain, palliative cancer pain, chemotherapy-induced nausea and vomiting, and spasticity related to multiple sclerosis or spinal cord injury, especially for those patients who do not respond to conventional therapies (Allan et al., 2018). Further randomized clinical trials that evaluate the efficacy and safety of $\mathrm{MC}$ in the management of CMP, other chronic pain or as substitute for opioids are urgently needed, but methodological challenges remain, including difficulties in participants' recruitment and follow-up, and the surveillance of adverse effects.

\section{Conclusion}

Although the included studies are frequently exploratory and might be biased by several factors, they describe the perspective of MC users and allow a better understanding of their attitudes and experiences regarding $\mathrm{MC}$ use against CMP and other chronic non-cancer pain. These users perceive $\mathrm{MC}$ to have more benefits than drawbacks regarding quality of life and adverse effects, and several report on the possibility that MC might decrease the use of some prescription drugs, particularly opioids. However, these user reported experiences must be examined by well-designed and methodologically sound clinical or observational studies before any conclusions can be drawn.

\section{Abbreviations}

CMP: Chronic musculoskeletal pain; MC: Medical cannabis; THC: Delta-9tetrahydrocannabinol; CBD: cannabidiol; NNT: Number needed to treat; HIV/ AIDS: Human immunodeficiency virus and acquired immune deficiency syndrome 


\section{Supplementary Information}

The online version contains supplementary material available at https://doi. org/10.1186/s42238-021-00096-8.

\section{Additional file 1.}

\section{Acknowledgements}

The authors are grateful to the pharmacists Norma Perez, MSc, and Rosa Martins, MSc., for their contribution during the screening of titles and abstracts and the review of eligible references.

\section{Authors' contributions}

DF performed part of the study selection and most of the synthesis of results and wrote subsequent drafts of the article; EK was the responsible supervisor for DF, wrote the protocol for the review, and was involved in al steps from the review to the writing of the article. MM and NJ both performed several steps of the review and commented on the article; $R B$, MW, GF-B, MA, and PP contributed to the research question at the origin of the review and to the writing at the article, while CED was involved in all steps of the review and the writing of the article and had the original research idea. The authors read and approved the final manuscript.

\section{Funding}

This study received financial support from the Centre de recherche sur les soins et les services de première ligne de l'Université Laval (Edeltraut Kröger and study team), from the Réseau québécois de recherche sur le vieillissement - FRQS, and the Centre de recherche sur le vieillissement de Québec of the Centre Intégré Universitaire de la Santé et de Services Sociaux de la Capitale Nationale (CIUSSSCN), as well as logistic support from the Centre de recherche du CHU de Québec - Université Laval (Edeltraut Kröger and part of the study team). None of the financial contributors participated in collection, analysis, or interpretation of data or in writing the manuscript.

\section{Availability of data and materials}

Not applicable.

\section{Declarations}

\section{Ethics approval consent to participate}

Not applicable.

\section{Consent for publication}

Not applicable.

\section{Competing interests}

Edeltraut Kröger, Daniela Furrer, Martine Marcotte, Nathalie Jauvin, Richard Bélanger, Guillaume Foldes-Busque, Michèle Aubin, Pierre Pluye and Clermont E. Dionne declare that they have no conflict of interest relevant to the content of this study. Mark Ware took a leave of absence from McGill University in 2018, well after most of this review was performed, and is now the Chief Medical Officer at Canopy Growth, Canada. We want to stress that at no time Dr. Ware's new affiliation influenced any step of this scoping review. Having been critically involved in research and in raising interest in the knowledge gap and growing request of patients to use cannabis for the treatment of chronic pain, he continues to be a collaborator in this research. Daniela Furrer has started to work at the Quebec Ministry of Health in July 2019.

\section{Author details}

${ }^{1}$ Centre d'excellence sur le vieillissement de Québec (CEVQ), Centre Intégré Universitaire de Santé et de Services Sociaux de la Capitale-Nationale (CIUSSSCN), Québec, QC, Canada. ${ }^{2}$ Centre de Recherche du CHU de Québec-Université Laval, Québec, QC, Canada. ${ }^{3}$ Faculty of Pharmacy, Université Laval, Québec, QC, Canada. ${ }^{4}$ Hôpital du Saint-Sacrement, 1050 Chemin Ste-Foy, room L2-30, Québec, QC G1S 4L8, Canada. ${ }^{5}$ Faculty of Medicine, Université Laval, Québec, QC, Canada. ${ }^{6}$ Faculty of Medicine, McGill University, Montréal, QC, Canada. 'School of Psychology, Université Laval, Québec, QC, Canada. ${ }^{8}$ Research Centre of the Centre Intégré de Santé et de Services Sociaux (CISSS) de Chaudière-Appalaches, Lévis, QC, Canada.
Received: 8 January 2021 Accepted: 10 August 2021

Published online: 04 September 2021

\section{References}

Abuhasira R, Shbiro L, Landschaft Y. Medical use of cannabis and cannabinoids containing products - regulations in Europe and North America. Eur J Intern Med. 2018;49:2-6. https://doi.org/10.1016/j.ejim.2018.01.001.

Aggarwal SK, Carter G, Sullivan M, Morrill R, Zumbrunnen C, Mayer J. Distress, coping, and drug law enforcement in a series of patients using medical cannabis. J Nerv Ment Dis. 2013a;201(4):292-303. https://doi.org/10.1097/ NMD.0b013e318288d333.

Aggarwal SK, Carter GT, Sullivan MD, ZumBrunnen C, Morrill R, Mayer JD. Characteristics of patients with chronic pain accessing treatment with medical cannabis in Washington State. J Opioid Manag. 2009;5(5):257-86. https://doi.org/10.5055/jom.2009.0028.

Aggarwal SK, Carter GT, Sullivan MD, Zumbrunnen C, Morrill R, Mayer JD. Prospectively surveying health-related quality of life and symptom relief in a lot-based sample of medical cannabis-using patients in urban Washington State reveals managed chronic illness and debility. Am J Hosp Palliat Med. 2013b;30(6):523-31. https://doi.org/10.1177/1049909112454215.

Aguilar S, Gutierrez V SL, Nougier M: Medicinal cannabis policies and practices around the world. In. London, UK; 2018: 32.

Alexandre CR. The Rhode Island medical marijuana program: an exploratory study. Policy Polit Nurs Pract. 2011;12(2):104-13. https://doi.org/10.1177/152 7154411403629.

Allan GM, Ramii J, Perry D, Ton J, Beahm NP, Crisp N, et al. Simplified guideline for prescribing medical cannabinoids in primary care. Can Fam Physician. 2018;64(2):111-20.

Arksey H, O'Malley L. Scoping studies: towards a methodological framework. Int J Soc Res Methodol. 2005;8(1):19-32. https://doi.org/10.1080/136455703200011 9616.

Arthritis Society: Arthritis in Canada. Facts \& Figures. In.: The Arthritis Society of Canada; 2015a: 1-8.

Arthritis Society: Research: Medical Cannabis. In Canada: The Arthritis Society; 2015b: 1-5.

Ashton CH. Pharmacology and effects of cannabis: a brief review. Br J Psychiatry. 2001;178(2):101-6. https://doi.org/10.1192/bjp.178.2.101.

Ashton JC. Cannabinoids for the treatment of inflammation. Curr Opin Investig Drugs. 2007;8(5):373-84.

Atluri S, Sudarshan G, Manchikanti L. Assessment of the trends in medical use and misuse of opioid analgesics from 2004 to 2011. Pain Physician. 2014; 17(2):E119-28.

Aviram J, Samuelly-Leichtag G. Efficacy of cannabis-based medicines for pain management: a systematic review and meta-analysis of randomized controlled trials. Pain Physician. 2017;20(6):E755-96.

Ballantyne JC. Opioid therapy in chronic pain. Phys Med Rehabil Clin N Am. 2015; 26(2):201-18. https://doi.org/10.1016/j.pmr.2014.12.001.

Beaulieu P, Boulanger A, Desroches J, Clark AJ. Medical cannabis: considerations for the anesthesiologist and pain physician. Can Anesth. 2016;63(5):608-24. https://doi.org/10.1007/s12630-016-0598-x.

Belle-Isle L, Walsh Z, Callaway R, Lucas P, Capler R, Kay R, et al. Barriers to access for Canadians who use cannabis for therapeutic purposes. Int I Drug Policy. 2014;25(4):691-9. https://doi.org/10.1016/j.drugpo.2014.02.009.

Bigand T, Anderson CL, Roberts ML, Shaw MR, Wilson M. Benefits and adverse effects of cannabis use among adults with persistent pain. Nurs Outlook. 2019;67(3):223-31. https://doi.org/10.1016/j.outlook.2018.12.014.

Blake DR, Robson P, Ho M, Jubb RW, McCabe CS. Preliminary assessment of the efficacy, tolerability and safety of a cannabis-based medicine (Sativex) in the treatment of pain caused by rheumatoid arthritis. Rheumatology (Oxford). 2006;45(1):50-2. https://doi.org/10.1093/rheumatology/kei183.

Boehnke KF, Litinas E, Clauw DJ. Medical Cannabis use is associated with decreased opiate medication use in a retrospective cross-sectional survey of patients with chronic pain. J Pain. 2016;17(6):739-44. https://doi.org/10.1016/ j.jpain.2016.03.002.

Bonn-Miller MO, Boden MT, Bucossi MM, Babson KA. Self-reported cannabis use characteristics, patterns and helpfulness among medical cannabis users. Am J Drug Alcohol Abuse. 2014;40(1):23-30. https://doi.org/10.3109/00952990.2 013.821477.

Bottorff JL, Bissell $\sqcup$, Balneaves LG, Oliffe JL, Kang HB, Capler NR, et al. Health effects of using cannabis for therapeutic purposes: a gender analysis of users' 
perspectives. Subst Use Misuse. 2011;46(6):769-80. https://doi.org/10.3109/1 0826084.2010.537732.

Bruce D, Brady JP, Foster E, Shattell M: Preferences for medical marijuana over prescription medications among persons living with chronic conditions: alternative, complementary, and tapering uses. J Alternative Complementary Med 2018, 24(2):146-153.

Brunt TM, van Genugten M, Honer-Snoeken K, van de Velde MJ, Niesink RJM. Therapeutic satisfaction and subjective effects of different strains of pharmaceutical-grade cannabis. J Clin Psychopharmacol. 2014;34(3):344-9. https://doi.org/10.1097/JCP.0000000000000129.

Burstein S. Cannabidiol (CBD) and its analogs: a review of their effects on inflammation. Bioorg Med Chem. 2015;23(7):1377-85. https://doi.org/10.1016/j.bmc.2015.01.059.

Carliner H, Mauro PM, Brown QL, Shmulewitz D, Rahim-Juwel R, Sarvet AL, et al. The widening gender gap in marijuana use prevalence in the U.S. during a period of economic change, 2002-2014. Drug Alcohol Depend. 2017;170:518. https://doi.org/10.1016/j.drugalcdep.2016.10.042.

Caulley L, Caplan B, Ross E. Medical marijuana for chronic pain. N Engl J Med. 2018;379(16):1575-7. https://doi.org/10.1056/NEJMclde1808149.

Colquhoun HL, Levac D, O'Brien KK, Straus S, Tricco AC, Perrier L, et al. Scoping reviews: time for clarity in definition, methods, and reporting. J Clin Epidemiol. 2014;67(12):1291-4. https://doi.org/10.1016/.j.clinepi.2014.03.013.

Coomber R, Oliver M, Morris C. Using cannabis therapeutically in the UK: a qualitative analysis. J Drug Issues. 2003;33(2):325-56. https://doi.org/10.1177/ 002204260303300204

Corroon JM Jr, Mischley LK, Sexton M. Cannabis as a substitute for prescription drugs - a cross-sectional study. J Pain Res. 2017;10:989-98. https://doi.org/1 $0.2147 / J P R . S 134330$.

Costa B, Trovato AE, Comelli F, Giagnoni G, Colleoni M. The non-psychoactive cannabis constituent cannabidiol is an orally effective therapeutic agent in rat chronic inflammatory and neuropathic pain. Eur J Pharmacol. 2007;556(13):75-83. https://doi.org/10.1016/j.ejphar.2006.11.006.

Cranford JA, Bohnert KM, Perron BE, Bourque C, Ilgen M. Prevalence and correlates of "Vaping" as a route of cannabis administration in medical cannabis patients. Drug Alcohol Depend. 2016;169:41-7. https://doi.org/10.1 016/j.drugalcdep.2016.10.008.

Crowell TL. Therapeutic value of medical marijuana in New Jersey patients: A community partnership research endeavor. J Allied Health. 2017;46(4):232-8.

de Meijer E: The chemical phenotypes (chemotypes) of cannabis. In: Handbook of Cannabis. edn. Edited by Pertwee RG: Oxford University Press; 2014.

Degenhardt L, Lintzeris N, Campbell G, Bruno R, Cohen M, Farrell M, et al. Experience of adjunctive cannabis use for chronic non-cancer pain: findings from the Pain and Opioids IN Treatment (POINT) study. Drug Alcohol Depend. 2015;147:144-50. https://doi.org/10.1016/j.drugalcdep.2014.11.031.

D'Souza DC, Ranganathan M. Medical Marijuana: is the cart before the horse? JAMA. 2015;313(24):2431-2. https://doi.org/10.1001/jama.2015.6407.

Elikottil J, Gupta P, Gupta K. The analgesic potential of cannabinoids. J Opioid Manag. 2009;5(6):341-57.

EISohly MA, Gul W. Constituents of Cannabis Sativa. In: Constituents of cannabis sativa. In: Handbook of Cannabis. edn. Edited by Pertwee RG. Oxford: Oxford University Press; 2014

Erkens JA, Janse AF, Herings RM. Limited use of medicinal cannabis but for labeled indications after legalization. Pharmacoepidemiol Drug Saf. 2005; 14(11):821-2. https://doi.org/10.1002/pds.1152.

Fanelli G, De Carolis G, Leonardi C, Longobardi A, Sarli E, Allegri M, et al. Cannabis and intractable chronic pain: an explorative retrospective analysis of Italian cohort of 614 patients. J Pain Res. 2017;10:1217-24. https://doi. org/10.2147/JPR.S132814

Fitzcharles MA, Baerwald C, Ablin J, Hauser W: Efficacy, tolerability and safety of cannabinoids in chronic pain associated with rheumatic diseases (fibromyalgia syndrome, back pain, osteoarthritis, rheumatoid arthritis) : a systematic review of randomized controlled trials. Schmerz 2016, 30(1):47-61, DOl: https://doi.org/10.1007/s00482-015-0084-3.

Gardiner KM, Singleton JA, Sheridan J, Kyle GJ, Nissen LM. Health professional beliefs, knowledge, and concerns surrounding medicinal cannabis - a systematic review. PLoS ONE. 2019;14(5):e0216556.

Gereau RW, Sluka KA, Maixner W, Savage SR, Price TJ, Murinson BB, et al. A pain research agenda for the 21st century. J Pain. 2014;15(12):1203-14. https://doi. org/10.1016/j.jpain.2014.09.004.

Goldenberg DL, Clauw DJ, Fitzcharles MA. New concepts in pain research and pain management of the rheumatic diseases. Semin Arthritis Rheum. 2011; 41(3):319-34. https://doi.org/10.1016/j.semarthrit.2011.04.005.
Gorter RW, Butorac M, Cobian EP, van der Sluis W. Medical use of cannabis in the Netherlands. Neurology. 2005;64(5):917-9. https://doi.org/10.1212/01.WNL. 0000152845.09088.28.

Grella CE, Rodriguez L, Kim T. Patterns of medical marijuana use among individuals sampled from medical marijuana dispensaries in los angeles. J Psychoactive Drugs. 2014;46(4):267-75. https://doi.org/10.1080/02791072.2 014.944960.

Grotenhermen F, Schnelle M. Survey on the medical use of Cannabis and THC in Germany. J Cannabis Therapeutics. 2003;3(2):17-40. https://doi.org/10.1300/J1 75v03n02_03.

Haroutounian S, Ratz Y, Ginosar Y, Furmanov K, Saifi F, Meidan R, et al. The effect of medicinal cannabis on pain and quality of life outcomes in chronic pain: a prospective open-label study. Clin J Pain. 2016;32(12):1036-43. https://doi. org/10.1097/AJP.0000000000000364.

Harris D, Jones RT, Shank R, Nath R, Fernandez E, Goldstein K, et al. Self-reported marijuana effects and characteristics of 100 San Francisco Medical Marijuana Club members. J Addict Dis. 2000;19(3):89-103. https://doi.org/10.1300/ J069v19n03_07.

Hauser W, Petzke F, Radbruch L, Tolle TR. The opioid epidemic and the long-term opioid therapy for chronic noncancer pain revisited: a transatlantic perspective. Pain Manag. 2016;6(3):249-63. https://doi.org/10.2217/pmt.16.5.

Hazekamp A, Heerdink ER. The prevalence and incidence of medicinal cannabis on prescription in The Netherlands. Eur J Clin Pharmacol. 2013;69(8):1575-80 https://doi.org/10.1007/s00228-013-1503-y.

Hazekamp A, Ware MA, Muller-Vahl KR, Abrams D, Grotenhermen F. The medicinal use of cannabis and cannabinoids--an international cross-sectional survey on administration forms. J Psychoactive Drugs. 2013;45(3):199-210. https://doi.org/10.1080/02791072.2013.805976.

Hoffman KA, Ponce Terashima J, McCarty D, Muench J. Toward a patient registry for cannabis use: an exploratory study of patient use in an outpatient healthcare clinic in Oregon. World Med Health Policy. 2017;9(3):307-17. https://doi. org/10.1002/wmh3.237.

Hosking RD, Zajicek JP. Therapeutic potential of cannabis in pain medicine. Br J Anaesth. 2008;101(1):59-68. https://doi.org/10.1093/bja/aen119.

Ilgen MA, Bohnert K, Kleinberg F, Jannausch M, Bohnert AS, Walton M, et al. Characteristics of adults seeking medical marijuana certification. Drug Alcohol Depend. 2013;132(3):654-9. https://doi.org/10.1016/j.drugalcdep.2 013.04.019.

Kilcher G, Zwahlen M, Ritter C, Fenner L, Egger M. Medical use of cannabis in Switzerland: analysis of approved exceptional licences. Swiss Med Wkly. 2017; 147:w14463.

Ko GD, Bober SL, Mindra S, Moreau JM. Medical cannabis - the Canadian perspective. J Pain Res. 2016;9:735-44. https://doi.org/10.2147/JPR.S98182.

Kosiba JD, Maisto SA, Ditre JW. Patient-reported use of medical cannabis for pain, anxiety, and depression symptoms: systematic review and meta-analysis. Soc Sci Med. 2019;233:181-92. https://doi.org/10.1016/j.socscimed.2019.06.005.

Lavie-Ajayi M, Shvartzman P. Restored self: a phenomenological study of pain relief by cannabis. Pain Med. 2018.

Levac D, Colquhoun H, O'Brien KK. Scoping studies: advancing the methodology. Implement Sci. 2010;5(69):69. https://doi.org/10.1186/1748-5908-5-69.

Lintzeris N, Driels J, Elias N, Arnold JC, MCGregor IS, Allsop DJ. Medicinal cannabis in Australia, 2016: the Cannabis as Medicine Survey (CAMS-16). Med J Aust. 2018;209(5):211-6. https://doi.org/10.5694/mja17.01247.

Lucas P, Walsh Z. Medical cannabis access, use, and substitution for prescription opioids and other substances: A survey of authorized medical cannabis patients. Int J Drug Policy. 2017;42:30-5. https://doi.org/10.1016/j.drugpo.201 7.01.011.

Lum HD, Arora K, Croker JA, Qualls SH, Schuchman M, Bobitt J, et al. Patterns of marijuana use and health impact: a survey among older Coloradans. Gerontology \& geriatric medicine. 2019;5:2333721419843707.

Lynch ME, Ware MA. Cannabinoids for the treatment of chronic non-cancer pain: an updated systematic review of randomized controlled trials. J Neuroimmune Pharmacol. 2015;10(2):293-301. https://doi.org/10.1007/s114 81-015-9600-6.

Lynch ME, Young J, Clark AJ. A case series of patients using medicinal marihuana for management of chronic pain under the Canadian Marihuana Medical Access Regulations. J Pain Symptom Manage. 2006;32(5):497-501. https://doi. org/10.1016/j.jpainsymman.2006.05.016.

MacCallum CA, Russo EB. Practical considerations in medical cannabis administration and dosing. Eur J Intern Med. 2018:49:12-9. https://doi.org/1 0.1016/j.jjim.2018.01.004. 
Maione S, Piscitelli F, Gatta L, Vita D, De Petrocellis L, Palazzo E, et al. Nonpsychoactive cannabinoids modulate the descending pathway of antinociception in anaesthetized rats through several mechanisms of action. Br J Pharmacol. 2011;162(3):584-96. https://doi.org/10.1111/j.1476-5381.2010. 01063.x.

McMahon SB, Koltzenburg M TI, Turk D: Wall \& Melzack's Textbook of Pain, 6th Edition. @Saunders: Elsevier; 2013.

Moore RA, Derry S, Taylor RS, Straube S, Phillips CJ. The costs and consequences of adequately managed chronic non-cancer pain and chronic neuropathic pain. Pain Pract. 2014;14(1):79-94.

National Academies of Sciences $E_{\text {, and Medicine: }}$ The health effects of cannabis and cannabinoids. The current state of evidence and recommendations for research. Washington DC: National Academies Press; 2017.

Niesink RJ, van Laar MW. Does cannabidiol protect against adverse psychological effects of THC? Front Psych. 2013;4:130.

Nugent SM, Morasco BJ, O'Neil ME, Freeman M, Low A, Kondo K, et al. The Effects of cannabis among adults with chronic pain and an overview of general harms: a systematic review. Ann Intern Med. 2017;167(5):319-31. https://doi.org/10.7326/M17-0155.

Nunberg H, Kilmer B, Pacula RL, Burgdorf J: An Nunberg H, Kilmer B, Pacula RL, Burgdorf J. An Analysis of Applicants Presenting to a Medical Marijuana Specialty Practice in California. J Drug Policy Anal. 2011;4(1):1. https://doi. org/10.2202/1941-2851.1017.

Ogborne AC, Smart RG, Weber T, Birchmore-Timney C. Who is using cannabis as a medicine and why: an exploratory study. J Psychoactive Drugs. 2000;32(4): 435-43. https://doi.org/10.1080/02791072.2000.10400245.

Park JY, Wu LT. Prevalence, reasons, perceived effects, and correlates of medical marijuana use: a review. Drug Alcohol Depend. 2017;177:1-13. https://doi. org/10.1016/j.drugalcdep.2017.03.009.

Pedersen ER, Hummer JF, Rinker DV, Traylor ZK, Neighbors C. Measuring protective behavioral strategies for marijuana use among young adults. J Stud Alcohol Drugs. 2016;77(3):441-50. https://doi.org/10.15288/jsad.2016.77.441.

Pedersen W, Sandberg S. The medicalisation of revolt: a sociological analysis of medical cannabis users. Sociol Health IIIn. 2013;35(1):17-32. https://doi.org/1 0.1111/j.1467-9566.2012.01476.x.

Perron BE, Bohnert K, Perone AK, Bonn-Miller MO, Ilgen M. Use of prescription pain medications among medical cannabis patients: comparisons of pain levels, functioning, and patterns of alcohol and other drug use. J Stud Alcohol Drugs. 2015;76(3):406-13. https://doi.org/10.15288/jsad.2015.76.406.

Petzke F, Enax-Krumova EK, Hauser W: [Efficacy, tolerability and safety of cannabinoids for chronic neuropathic pain: a systematic review of randomized controlled studies]. Schmerz 2016, 30(1):62-88, DOl: https://doi. org/10.1007/s00482-015-0089-y.

Piper BJ, Beals ML, Abess AT, Nichols SD, Martin MW, Cobb CM, et al. Chronic pain patients' perspectives of medical cannabis. Pain. 2017;158(7):1373-9. https://doi.org/10.1097/j.pain.0000000000000899.

Podichetty VK, Mazanec DJ, Biscup RS. Chronic non-malignant musculoskeletal pain in older adults: clinical issues and opioid intervention. Postgrad Med J. 2003;79(937):627-33. https://doi.org/10.1136/pmj.79.937.627.

Reiman A. Medical cannabis patients: patient profiles and health care utilization patterns. Complementary Health Practice Review. 2007;12(1):31-50. https:// doi.org/10.1177/1533210107301834.

Reiman A. Cannabis as a substitute for alcohol and other drugs. Harm Reduct J. 2009;6(1):35. https://doi.org/10.1186/1477-7517-6-35.

Reiman A, Welty M, Solomon P. Cannabis as a Substitute for Opioid-Based Pain Medication: Patient Self-Report. Cannabis Cannabinoid Res. 2017;2(1):160-6. https://doi.org/10.1089/can.2017.0012.

Reinarman C, Nunberg H, Lanthier F, Heddleston T. Who are medical marijuana patients? Population characteristics from nine California assessment clinics. J Psychoactive Drugs. 2011:43(2):128-35. https:/doi.org/10.1080/02791072.2011.587700.

Rowe J, Caprio AJ. Chronic pain: challenges and opportunities for relieving suffering. N C Med J. 2013;74(3):201-4. https://doi.org/10.18043/ncm.74.3.201.

Sagy I, Bar-Lev Schleider L, Abu-Shakra M, Novack V: Safety and efficacy of medical cannabis in fibromyalgia. J Clin Med 2019, 8(6).

Schaefert R, Welsch P, Klose P, Sommer C, Petzke F, Hauser W. Opioids in chronic osteoarthritis pain. A systematic review and meta-analysis of efficacy, tolerability and safety in randomized placebo-controlled studies of at least 4 weeks duration. Schmerz. 2015;29(1):47-59. https://doi.org/10.1007/s00482-014-1451-1.

Schnelle M, Grotenhermen F, Reif M, Gorter RW: [Results of a standardized survey on the medical use of cannabis products in the German-speaking area]. Forschende Komplementarmedizin 1999, 6(Suppl 3):28-36.
Sexton M, Cuttler C, Finnell JS, Mischley LK. A Cross-sectional survey of medical cannabis users: patterns of use and perceived efficacy. Cannabis Cannabinoid Res. 2016;1(1):131-8. https://doi.org/10.1089/can.2016.0007.

Shah A, Craner J, Cunningham JL. Medical cannabis use among patients with chronic pain in an interdisciplinary pain rehabilitation program: characterization and treatment outcomes. J Subst Abuse Treat. 2017;77:95100. https://doi.org/10.1016/j.jsat.2017.03.012.

Shiplo S, Asbridge M, Leatherdale ST, Hammond D. Medical cannabis use in Canada: vapourization and modes of delivery. Harm Reduct J. 2016;13(1):30. https://doi.org/10.1186/s12954-016-0119-9.

Skrabek RQ, Galimova L, Ethans K, Perry D. Nabilone for the treatment of pain in fibromyalgia. J Pain. 2008;9(2):164-73. https://doi.org/10.1016/j.jpain.2007.09. 002.

Ste-Marie PA, Fitzcharles MA, Gamsa A, Panopalis P, Shir Y. Cannabinoid use in fibromyalgia is associated with male gender, opioid use and drug seeking behaviour. Pain Res Manag. 2012;17(3):224.

Ste-Marie PA, Shir Y, Rampakakis E, Sampalis JS, Karellis A, Cohen M, et al. Survey of herbal cannabis (marijuana) use in rheumatology clinic attenders with a rheumatologist confirmed diagnosis. Pain. 2016;157(12):2792-7. https://doi. org/10.1097/j.pain.0000000000000706.

Stockings E, Campbell G, Hall WD, Nielsen S, Zagic D, Rahman R, et al. Cannabis and cannabinoids for the treatment of people with chronic noncancer pain conditions: a systematic review and meta-analysis of controlled and observational studies. Pain. 2018;159(10):1932-54. https://doi.org/10.1097/j.pa in.0000000000001293.

Swift W, Gates P, Dillon P. Survey of Australians using cannabis for medical purposes. Harm Reduct J. 2005;2(1):18. https://doi.org/10.1186/1477-7517-2-1 8.

Task Force on Taxonomy of the International Association for the Study of Pain: Classification of chronic pain. Descriptions of chronic pain syndromes and definition of pain terms. In. Seattle, USA: International Association for the Study of Pain (IASP); 1994

Tobin DG, Andrews R, Becker WC. Prescribing opioids in primary care: Safely starting, monitoring, and stopping. Cleve Clin J Med. 2016;83(3):207-15. https://doi.org/10.3949/ccjm.83a.15034.

Troutt WD, DiDonato MD. Medical cannabis in Arizona: patient characteristics, perceptions, and impressions of medical cannabis legalization. J Psychoactive Drugs. 2015;47(4):259-66. https://doi.org/10.1080/02791072.2015.1074766.

Walsh Z, Callaway R, Belle-Isle L, Capler R, Kay R, Lucas P, et al. Cannabis for therapeutic purposes: patient characteristics, access, and reasons for use. Int J Drug Policy. 2013;24(6):511-6. https://doi.org/10.1016/j.drugpo.2013.08.010.

Ware MA, Adams H, Guy GW. The medicinal use of cannabis in the UK: results of a nationwide survey. Int J Clin Pract. 2005;59(3):291-5. https://doi.org/1 0.1111/j.1742-1241.2004.00271.x.

Ware MA, Doyle CR, Woods R, Lynch ME, Clark AJ. Cannabis use for chronic noncancer pain: results of a prospective survey. Pain. 2003;102(1-2):211-6. https:// doi.org/10.1016/s0304-3959(02)00400-1.

Webb CW, Webb SM. Therapeutic benefits of cannabis: a patient survey. Hawai'i J Med Public Health. 2014;73(4):109-11.

Whiting PF, Wolff RF, Deshpande S, Di Nisio M, Duffy S, Hernandez AV, et al. Cannabinoids for medical use: a systematic review and meta-analysis. JAMA. 2015;313(24):2456-73. https://doi.org/10.1001/jama.2015.6358.

Woolf AD, Erwin J, March L. The need to address the burden of musculoskeletal conditions. Best Pract Res Clin Rheumatol. 2012;26(2):183-224. https://doi. org/10.1016/j.berh.2012.03.005.

Yelin E, Weinstein S, King T. The burden of musculoskeletal diseases in the United States. Semin Arthritis Rheum. 2016;46(3):259-60. https://doi.org/10.1 016/j.semarthrit.2016.07.013.

Zaller N, Topletz A, Frater S, Yates G, Lally M. Profiles of medicinal cannabis patients attending compassion centers in Rhode Island. J Psychoactive Drugs. 2015;47(1):18-23. https://doi.org/10.1080/02791072.2014.999901.

Zhu HJ, Wang JS, Markowitz JS, Donovan JL, Gibson BB, Gefroh HA, et al. Characterization of P-glycoprotein inhibition by major cannabinoids from marijuana. J Pharmacol Exp Ther. 2006;317(2):850-7. https://doi.org/10.1124/jpet.105.098541.

Zolotov Y, Baruch Y, Reuveni H, Magnezi R. Adherence to Medical cannabis among licensed patients in Israel. Cannabis Cannabinoid Res. 2016;1(1):16-21. https://doi.org/10.1089/can.2015.0003.

\section{Publisher's Note}

Springer Nature remains neutral with regard to jurisdictional claims in published maps and institutional affiliations. 